\title{
Design studies and multi-disciplinary assessment of agile and highly swept flying wing configurations
}

\author{
Carsten M. Liersch ${ }^{1} \oplus \cdot$ Andreas Schütte $^{1} \cdot$ Martin Siggel $^{2} \cdot$ Jochen Dornwald $^{3}$
}

Received: 28 February 2019 / Revised: 16 September 2019 / Accepted: 18 April 2020 / Published online: 18 May 2020

(c) The Author(s) 2020

\begin{abstract}
This article belongs to a series of publications on the design and assessment of the MULDICON UCAV configuration. MULDICON is a $53^{\circ}$ swept flying wing combat aircraft concept, investigated in a common effort between the DLR research project Mephisto and the NATO STO Research Task Group AVT-251. This article focuses on the development of the overall concept starting from its predecessor, the SACCON configuration, and the requirements which had been defined for the MULDICON design task. A second focus is placed on the investigation of the vortex-dominated flow topology arising around such a configuration and its sensitivity to specific geometric changes. Furthermore, the article briefly introduces the DLR conceptual aircraft design system and the extensions which have been developed for improving its capabilities for UCAV assessment. Finally, an industrial view on the MULDICON design task is provided, as well as a conclusion of the Mephisto results and an outlook upon further work.
\end{abstract}

Keywords Conceptual aircraft design $\cdot$ Flying wing $\cdot$ Vortex-dominated flow $\cdot$ Mephisto $\cdot$ SACCON $\cdot$ MULDICON

$\begin{array}{ll}\text { Abbreviations } \\ \text { AVT } & \text { Applied vehicle technology } \\ \text { CAD } & \text { Computer-aided design } \\ \text { CG } & \text { Center of gravity } \\ \text { CPACS } & \begin{array}{l}\text { Common parametric aircraft configuration } \\ \text { schema }\end{array} \\ \text { CoC } & \text { Center of competence } \\ \text { CST } & \text { Class-shape function transformation } \\ \text { DLR } & \text { Deutsches Zentrum für Luft- und } \\ & \text { Raumfahrt } \\ \text { FaUSST } & \text { DLR research project 2011-2013 } \\ \text { MAC } & \text { Mean aerodynamic chord } \\ \text { Mephisto } & \text { DLR research project, 2014-2018 } \\ \text { MIL-STD } & \text { United States Military Standard } \\ \text { MRP } & \text { Moment reference point } \\ \text { MTOM } & \text { Maximum takeoff mass } \\ \text { MULDICON } & \text { Multi-disciplinary configuration }\end{array}$

Carsten M. Liersch

Carsten.Liersch@dlr.de

1 DLR, Institute of Aerodynamics and Flow Technology, Lilienthalplatz 7, 38108 Braunschweig, Germany

2 DLR, Simulation and Software Technology, Linder Höhe, 51147 Köln, Germany

3 Airbus Defence \& Space, Rechliner Straße, 85077 Manching, Germany
NACA National advisory committee for aeronautics

NATO

OEM

RANS

RCE

SACCON

STO

TiGL

TiXI

UCAV

UCAV-2010

XML

ZFM

$A, B, C$

$C_{D}$

$C_{L}$

$C_{m}$

$C_{p}$

$C_{T}$

deg

$I_{x x}, I_{y y}, I_{z z}$

$n_{z}$
North-Atlantic Treaty Organization

Operational empty mass

Reynolds averaged Navier-Stokes equations

Remote component environment

Stability and control configuration

NATO Science and Technology

Organization

TiGL Geometry Library

TiXI XML interface

Unmanned combat aerial vehicle

DLR research project, 2007-2010

EXtensible markup language

Zero fuel mass

Specific sections of the wing

Drag force coefficient

Lift force coefficient

Pitching moment coefficient

Pressure coefficient

Thrust force coefficient

Degree of arc (also written as ${ }^{\circ}$ )

Mass moments of inertia around $X, Y, Z$

Vertical load factor 
$r$

$X, Y, Z$

Relative airfoil leading edge curvature radius

$\alpha$ Coordinates

$\varepsilon_{\mathrm{LE}}, \varepsilon_{\mathrm{TE}}$ Angle of attack

Twist angle around the leading/trailing edge

$\varphi_{\mathrm{LE}}$

Sweep angle of the leading edge

\section{Introduction}

The design of agile and highly swept flying wing $U C A V$ configurations is a challenging task: The assessment of performance and maneuverability requirements, which are essential design drivers for the overall concept, demands for rather detailed data concerning mass distribution, engine performance, and aerodynamic characteristics for a wide range of flight conditions. The classical aerodynamic models used within conceptual aircraft design are based upon potential flow theory. Generally speaking, they are fast and robust, but their ability to predict transonic behavior and the nonlinearity due to flow separation and vortex effects is rather limited. Thus, for the design of agile aircraft configurations where the flow field is dominated by vortex effects triggered from small geometrical details, it is inevitable to use highfidelity, RANS-based aerodynamic methods, as well.

Such a design case was investigated within the DLR research project Mephisto. Its aim was to redesign the SACCON UCAV configuration, known from its predecessor projects UCAV-2010 and FaUSST [1], with respect to a set of extended, agility-related design requirements. For this enhanced concept, the name MULDICON was chosen.

This article is focused on the design of the MULDICON configuration, which was performed in close cooperation with the NATO STO task group AVT-251 on "Multi-Disciplinary design and performance assessment of effective, agile NATO Air Vehicles" [2]. It belongs to a series of Mephisto-based publications, covering various aspects of the SACCON redesign task. As a starting point, Chapter 2 provides some background information and history of SAC$\mathrm{CON}$, as well as the design requirements which were specified for MULDICON. Chapter 3 is dedicated to the design methodology and the enhancements of the DLR design system which were introduced within Mephisto. To get a better understanding of the occurring vortex effects and to learn how to influence and control them, a great amount of aerodynamic design studies have been conducted using highfidelity RANS methods. Some of these studies are discussed in Chapter 4. The new MULDICON concept is finally presented in Chapter 5. Chapter 6 focuses on the industrial perspective and the benefit, the presented methodology offers for industrial application. Concluding this article, a final

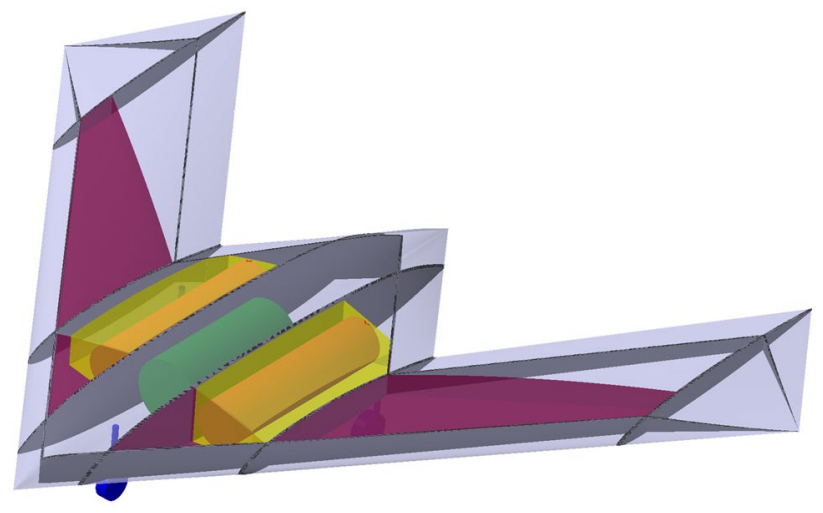

Fig. 1 SACCON UCAV concept

summary and an outlook towards future work are provided in Chapter 7.

\section{Design task}

A central part of the DLR research project Mephisto was the design of the MULDICON configuration based on its predecessor concept SACCON. In the first part of this chapter, a brief overview of SACCON and its history is provided. The second part of the chapter contains a description of the specifications and design requirements which were selected for the MULDICON design task.

\subsection{Background}

The starting point for the MULDICON design work was the so-called SACCON configuration, a tailless, lambda-shaped flying wing UCAV concept, characterized by a $53^{\circ}$ swept wing with parallel edges for low radar signature purposes. It is a common research concept exposing complex, vortexdominated flow structures which are highly challenging to predict. SACCON was defined in a common effort of the DLR research project UCAV-2010 and the NATO STO Task Group AVT-161 on "Assessment of Stability and Control Prediction Methods for NATO Air and Sea Vehicles" [3, 4].

Initially SACCON was a pure outer shape, serving as a benchmark for CFD methods and wind tunnel experiments. Within the DLR successor project FaUSST (and together with the NATO STO task group AVT-201 on "Extended Assessment of Stability and Control Prediction Methods for NATO Air Vehicles"), an attempt was made to turn the SACCON shape into a reasonable aircraft concept. Therefore, a set of requirements were defined, including a design mission to be flown, a payload to be carried, and some further parameters like fuel reserve and stability margin. The internal arrangement (see Fig. 1) with a single, central engine 
Table 1 Main design parameters of MULDICON

\begin{tabular}{|c|c|}
\hline Parameter & Value \\
\hline Outer shape & $\begin{array}{l}\text { Based on SACCON, } \\
\pm 30^{\circ} \text { trailing edge sweep }\end{array}$ \\
\hline Propulsion & Single turbofan engine without afterburner \\
\hline Propulsion integration & Internal (due to signature reasons) \\
\hline Static dry thrust & Thrust-to-weight ratio $=0.4(\approx 60 \mathrm{kN})$ \\
\hline Payload storage & Internal (due to signature reasons) \\
\hline Payload bay size & $\begin{array}{l}\text { Length: } 4.2 \mathrm{~m} \\
\text { Width: } 1.0 \mathrm{~m}\end{array}$ \\
\hline Payload mass & $2 \times 1250 \mathrm{~kg}$ \\
\hline Design range & $3000 \mathrm{~km}$ (without aerial refueling) \\
\hline Fuel reserve & $\approx 45 \mathrm{~min}$ \\
\hline Cruise altitude & $11 \mathrm{~km}$ \\
\hline Cruise Mach number & 0.8 (all altitudes) \\
\hline Stability margin & 0-3\% MAC (stable) \\
\hline CG range & $5.82 \mathrm{~m}-6.00 \mathrm{~m}$ \\
\hline
\end{tabular}

(shown in green) and two payload/weapon bays aside (shown in yellow) offers the best opportunity for an efficient propulsion with a low SFC. To limit the center of gravity $(\mathrm{CG})$ movement due to fuel consumption, a concept with two fuel tanks (shown in red) on each side which are having a common $\mathrm{CG}$ within the specified $\mathrm{CG}$ range of the aircraft was chosen. The complete design process, as well as the final concept, is described in detail in Refs. [1, 5-7].

Even though a basic mission capability could be reached for the SACCON concept, several unsatisfactory aspects remained, indicating a necessity for significant changes in the SACCON outer shape. Together with some additional agility requirements, these unsatisfactory aspects were used to define the requirements catalog for the design of MULDICON.

\subsection{MULDICON design requirements}

The rationale behind the development of the MULDICON concept was to overcome the known deficiencies of SAC$\mathrm{CON}$ and to evolve it into a controllable and agile UCAV configuration which is consistent from a conceptual aircraft design point of view. To stay as close to the SACCON concept as possible, it was agreed that most of the requirements from SACCON should remain the same and that the changes of the outer shape should be limited to a minimum. The following sections briefly discuss the various design requirements; Table 1 provides a quick overview of the most important aspects. Some further details and thoughts behind the design requirements are published by Liersch and Bishop [8].

\subsubsection{Design mission}

The design mission for MULDICON was taken from SACCON without any change. Altitude and Mach number are sketched over distance to the starting point (from left to right and back to the left) in Fig. 16 in the appendix.

It is a rather classical long range transport mission with a radius of $1500 \mathrm{~km}$ and no aerial refueling, which is closely related to "Bomber-low-level penetration" from the United States Military Standard MIL-STD-3013 [9]. It consists of a high altitude cruise segment, followed by a low altitude dash approaching the target. After passing the target, it continues with a turn and returns back to the starting base with the same flight profile. Nearly all the mission is flown with a Mach number of 0.8 - except for the initial climb/final descent and the turn after passing the target. During the last $75 \mathrm{~km}$ before reaching the target area, the speed could be further increased up to a Mach number of 0.9. However, this acceleration is just an option to exploit existing performance reserves of the aircraft and not a design requirement itself. The high-level cruise flight segment is performed at an altitude of $11 \mathrm{~km}$, the low-level dash when approaching and leaving the target is started at an altitude of $1000 \mathrm{~m}$, descending to $300 \mathrm{~m}$ and climbing back to $1000 \mathrm{~m}$ on the way back. An additional fuel reserve of approximately 45 min of flight time is desired.

In addition to the basic mission as it was specified for SACCON, maneuver agility and other requirements for each section of the mission were defined, mostly in dependence on the United States Military Standard MIL-STD-1797A [10]. ${ }^{1}$

\subsubsection{Payload}

As for SACCON, it was decided for MULDICON to use a configuration layout with one central engine and payload/ weapon bays on either side. To determine the required size of the bays and the maximum payload mass to be carried, a weapon systems study was performed. With respect to different possible weapons (which are not listed in detail here), a payload/weapon bay length of $4.2 \mathrm{~m}$ and a width of $1.0 \mathrm{~m}$ was chosen. The height of the bay depends on the outer shape and the structural concept of the aircraft. The maximum payload mass for each bay has been agreed to be $1250 \mathrm{~kg}$. The payload/weapon bays shall be located within the permitted CG range (see Sect. 2.2.4) to minimize CG movement due to payload drop/weapon release.

\footnotetext{
${ }^{1}$ Even though MULDICON is not a piloted aircraft, many of the requirements from this standard seem to be appropriate here, as well.
} 


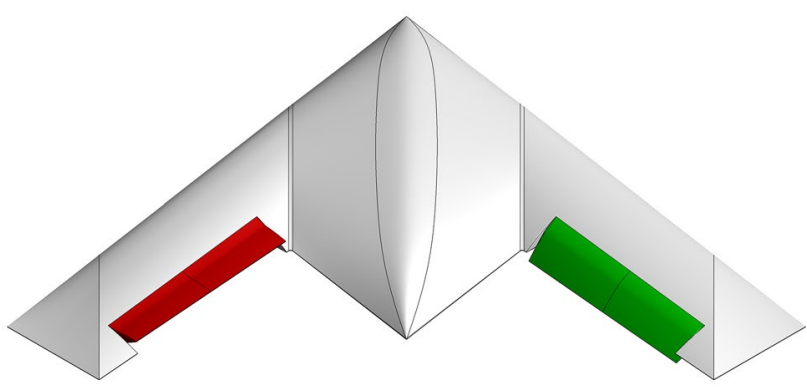

Fig. 2 SACCON Control Surfaces (taken from [12])

\subsubsection{Agility and control concept}

With respect to the demand of evolving SACCON into a controllable, agile UCAV, there are several new requirements coming from the field of stability and control. First of all, SACCON is lacking a reasonable control concept. It is equipped with two trailing edge control surfaces on each side of the wing for roll and pitch control, as can be seen in Fig. 2. During the investigations of AVT-201, it turned out that — due to the high trailing edge sweep of the wing - these control surfaces exhibit very poor performance compared to the theoretical optimum (for which the simple aerodynamic methods used in conceptual design are a coarse prediction) $[1,6,11]$.

Considering yaw control, several different flap and spoiler concepts were studied for SACCON [13], but none of these performed well. Again, the main reason for the limited control effectiveness seems to have its roots in the high trailing edge sweep of the wing.

As a consequence of these control problems, it was agreed within Mephisto and AVT-251 to reduce the trailing edge sweep from $53^{\circ}$ (as it was for SACCON) to $30^{\circ}$. Even though this certainly adds additional peaks to its radar signature, it was assumed that the performance of the trailing edge devices would be enhanced and that the modified flow would also enable an appropriate yaw control concept.

To define agility requirements for MULDICON, five dedicated design points covering various flight conditions were selected and specified. Even though there are already agility requirements defined for each section of the design mission (as described in Sect. 2.2.1), with respect to available resources, it was agreed to focus on these five points only. Table 5 in the appendix lists the flight conditions for the design points and the corresponding requirements for MULDICON.

Each design point consists of altitude, velocity, mass, and vertical load factor. There are two mass cases: $15000 \mathrm{~kg}$ (Design maximum takeoff mass (MTOM)) and $13000 \mathrm{~kg}$ (Design MTOM, after use of 1/3 of maximum fuel). The vertical load factors can be seen as pull-up or turn requirements.
In case of performing a sustained turn ${ }^{2}$ with the defined load factor, an approximation for the corresponding turn radius is provided, as well. The last entry under "Flight conditions" contains an assumption for the lift-to-drag ratio in that flight point (including load factor). The "Design requirements" part of the table contains first the specified roll, pitch and yaw requirements. These are either given for a steady rotation rate in degrees per second, or as a performance requirement to reach a certain angle within a specified time, starting from steady, horizontal flight. Aside from the specified yaw rate, there are two further main aspects which are crucial for sizing the yaw control concept: the ability to apply yawing moments rapidly to compensate the expected directional instability of MULDICON and the so-called "de-crab" maneuver for landing under maximum permitted crosswind conditions. The requirement on permitted crosswind for takeoff and landing is again taken from MIL-STD1797A [10] and provided in the next line of the table. The lift coefficients in the following line are necessary to fly in that point and with the given load factor; the maximum lift coefficients (which are the same values, increased by a safety margin of 0.1 ) are the requirements for the aerodynamic shape design: MULDICON shall be able to reach that maximum lift coefficient under the specified flight conditions. To provide an idea of the required changes compared to SACCON, the subsequent line gives an assumption of the maximum lift coefficients SACCON reaches for the same cases. Using lift coefficient and lift-to-drag ratio from above, the assumed drag coefficient and the required thrust for a sustained turn are calculated and placed in the last two lines. These are essential requirements for the engine design work. It has to be mentioned that for the "Combat High Altitude" case a sustained turn requirement was not specified: Under such flight conditions, a sustained turn with a load factor of 4.5 would not be reasonable with respect to the selected engine concept ${ }^{3}$ (see Sect. 2.2.5).

\subsubsection{CG range and pitching moment characteristic}

From SACCON, it is already known that development, movement, and interaction of vortices with increasing angle of attack severely influence the pitching moment characteristics. These effects were investigated in detail by Schütte et al. [14] and lead to a diagram which is shown in Fig. 3. ${ }^{4}$

\footnotetext{
2 A "sustained turn" is a turn without changing altitude or speed.

${ }^{3}$ For that combination of velocity, altitude and load factor, the required thrust would be around $83 \mathrm{kN}$.

${ }^{4}$ In order to understand this diagram correctly, it has to be mentioned that the moment reference point of SACCON is located far behind the permitted CG range. Thus, the positive (pitch-up) gradient for lower angles of attack does not mean that the aircraft is unstable there.
} 


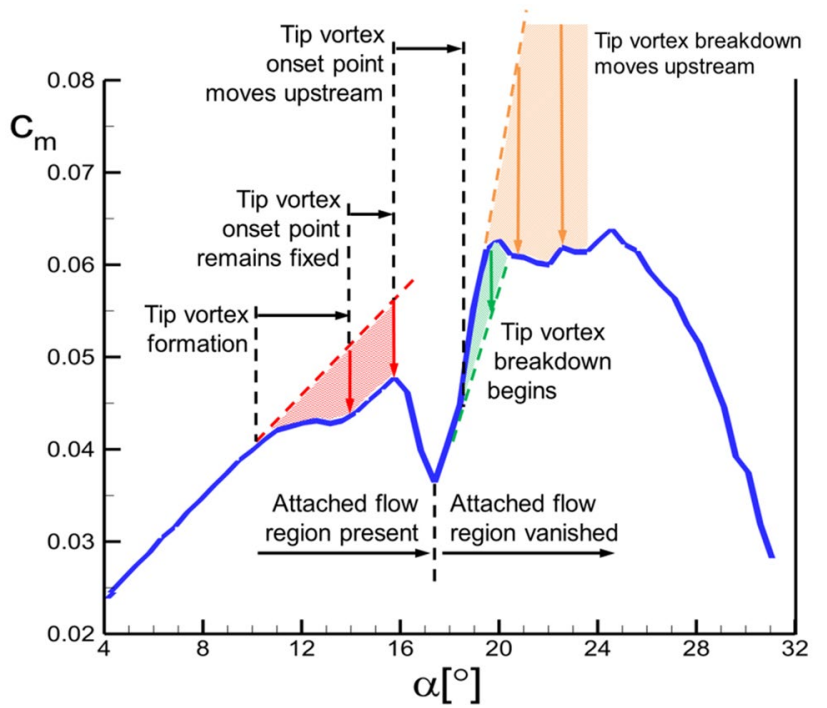

Fig. 3 SACCON pitching moment curve (taken from [14])

Looking at the moment curve of SACCON, it becomes apparent that such a nonlinear characteristic is not acceptable for a combat aircraft operating at angles of attack up to $20^{\circ}$ or even beyond. Thus, one central task for aerodynamic shaping was to modify the outer shape in such a way that the pitching moment characteristic becomes much smoother.

To increase the agility of MULDICON around the pitch axis (compared to SACCON), the permitted CG range was reduced down from $2-8 \%$ of the mean aerodynamic chord (MAC) (SACCON) to 0-3\% MAC (MULDICON) on the stable side of the neutral point. ${ }^{5}$ After performing some first computations, the neutral point was approximated to lie $6 \mathrm{~m}$ behind the nose of the aircraft. This neutral point is also taken as moment reference point for the aerodynamic investigations. Together with a MAC length of $6 \mathrm{~m}$ (which is taken as reference length for normalizing all three moment coefficients, as well), this leads to a permitted CG range between 5.82 and $6 \mathrm{~m}$, counted from the nose of the aircraft.

\subsubsection{Propulsion}

For efficiency reasons, the MULDICON configuration shall be designed with a single, central engine without afterburner (see Sect. 2.1). Based on experience from SACCON, the static dry thrust of the engine shall correspond to a thrustto-weight ratio of 0.4 ; for an estimated MTOM of 15 metric tons, this leads a thrust value of $60 \mathrm{kN}$. The engine shall be

\footnotetext{
5 Due to the vortex-dominated flow topology, there is not one single neutral point. However, with respect to the design requirement of smoothing the pitching moment curve, it is still reasonable to assume an average position for it.
}

optimized for cruise performance (see design point "Cruise" in Table 5); however, its fan diameter shall not exceed $1 \mathrm{~m}$. On top of these requirements, which are rather similar to those of SACCON, the MULDICON engine needs to deliver sufficient thrust for performing the sustained turn maneuvers that are described above in Sect. 2.2.3.

\section{Conceptual design methodology}

After specifying and agreeing the design requirements together with the partners from Mephisto and AVT-251, the conceptual aircraft design work at DLR was started.

\subsection{DLR design system and extensions}

First, the DLR conceptual aircraft design system [15-17] was used to create a suitable analysis process. It is a flexible concept design toolset being developed at DLR since 2005, connecting analysis tools from different disciplines and the corresponding experts at the different DLR sites in Germany. Recent applications of the system can be found in [18-20]. To understand the analysis process being created and applied for the design of MULDICON, the DLR conceptual aircraft design system is introduced first. It consists of three main components.

\section{- Data exchange}

A data exchange file format called CPACS is being developed for the DLR aircraft design system [21]. CPACS is an XML-based data format which is designed to store aircraft data and geometries in a hierarchical and parametric way. It was introduced mainly to serve as a common language between the different disciplines' analysis tools. Two software libraries [22] called TiXI and TiGL are being developed to ease the use of CPACS. While TiXI provides a simple interface to create, read, modify, and write XML datasets such as CPACS, TiGL generates a 3D CAD model of the aircraft from the parametric data and offers methods to query geometric data from this model. In addition, TiGL provides functions to store the generated geometry using standard CAD exchange file formats. The TiGL Viewer application can be used to visualize the underlying CAD model. The complete package of CPACS and libraries is available under open source licenses [23-25].

\section{- Analysis tools}

The analysis of an aircraft configuration is performed by disciplinary analysis tools which are provided and maintained by the discipline experts. For many disciplines, there is already more than one tool available—each one covers a 
different level of fidelity or uses a different way of modeling. What they all have in common is the need to read and write CPACS datasets as input and output. For new tools, it is certainly a good way to use the CPACS data format directly. For legacy codes, which typically have (and shall keep) their own data formats, the best way is to use a so-called "toolwrapper". A toolwrapper is a small program which reads a CPACS file, writes an input file for the tool, runs the tool, reads the output file of the tool and finally writes the results as a CPACS file. To keep the disciplinary experts in the loop and to avoid a decoupling of the used tools from further development, the tools are not gathered at one location to form a monolithic program. Instead, they are placed on disciplinary tool servers which stay under maintenance and supervision of the corresponding experts. Using a software integration framework, these distributed tools can be plugged together to form process chains for aircraft design and analysis.

\section{- Integration framework}

The software integration framework serves as a sort of construction kit. Here, the disciplinary tools, which are located on distributed servers, can be linked together to create process chains, customized for individual design or analysis tasks. Trade-study tools, different optimizers and other drivers of the process may be applied to get an impression of the sensitivities of the design parameters, as well as optimal solutions for specific target functions. For SACCON design, still the commercial ModelCenter [26] framework software was used to build the analysis workflows. However, during the last years the DLR in-house software RCE [27] has replaced ModelCenter as central integration framework for conceptual aircraft design. In line with CPACS, TiXI, and TiGL, RCE is provided to the public under an open source license, as well [28].

During Mephisto, the DLR design system was extended in several ways. To simulate the agility and control concept, the TiGL geometry generator had to be extended to integrate control surfaces into its CAD model. This enables to model control surfaces at different geometrical fidelities, starting with a simple cutout of the wing up to precise modeling of the flap profiles, including the gaps. Although only trailing edge devices need to be modeled for the MULDICON configuration, support for leading edge devices has also been included in the TiGL software. In addition to aerodynamic design studies, TiGL also had to support structural and aeroelastic analyses. Therefore, TiGL has also been extended to model spars and ribs according to their CPACS definition. This was originally developed by Airbus Defense and Space in a separate, customized version of TIGL, but the code was finally reintroduced to the open source TiGL code base.

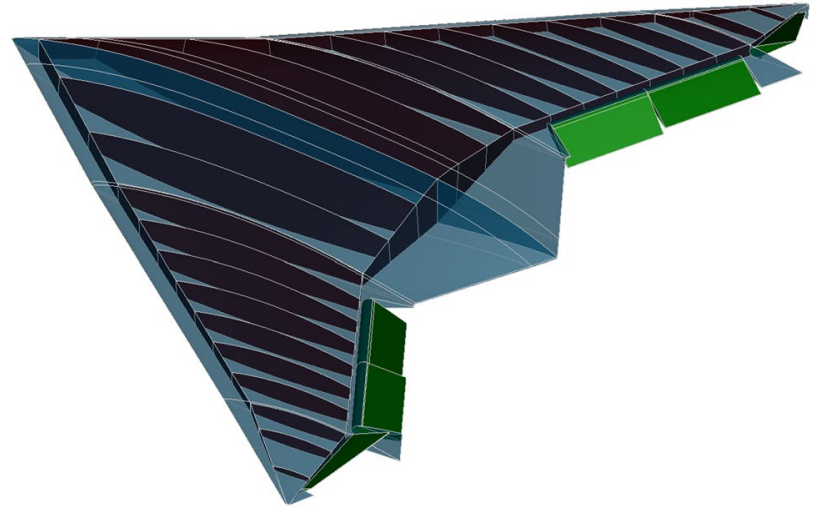

Fig. 4 TiGL model of MULDICON with deflected control surfaces and simplified structure

Figure 4 shows the TiGL model of MULDICON including the newly added control surfaces ${ }^{6}$ and the wing structure. ${ }^{7}$

In addition to the changes required to model MULDICON, the CPACS 3 format was developed. CPACS 3 solves many problems that have often arisen with CPACS 2, especially in the area of the wing geometry. Accordingly, TiGL and TiXI had to be adapted to CPACS 3.

The RCE integration framework has been adapted for the use of very large workflows. It is now possible to use nested loops in RCE, e.g. to implement coupled simulations inside an optimization loop. It is also now possible to define how RCE should behave in case of an error in a tool. Therefore, RCE can try to start the tool several times or ignore the output and continue the optimization with new design parameters. The caching of intermediate results has also been implemented in RCE. This simplifies the debugging of workflows considerably, since time-consuming computations are simply skipped after a restart of the whole workflow.

\subsection{MULDICON design process}

Based on CPACS, RCE, and selected analysis modules a workflow for investigating and sizing MULDICON was created (see workflow diagram in Fig. 5). It consists of three consecutive steps which are marked in red, blue and green. Each of the big squares in the diagram represents one of the distributed analysis tools which have been selected for this process. The arrows indicate the way how data are distributed in the workflow. Typically, such an arrow stands for

\footnotetext{
${ }^{6}$ As CPACS does not yet permit to specify split flaps, ordinary trailing edge flaps at the wingtips are used instead.

7 In this case, a simplified structural model containing only two main spars and no cutouts for engine or payload/weapon bays is shown.
} 


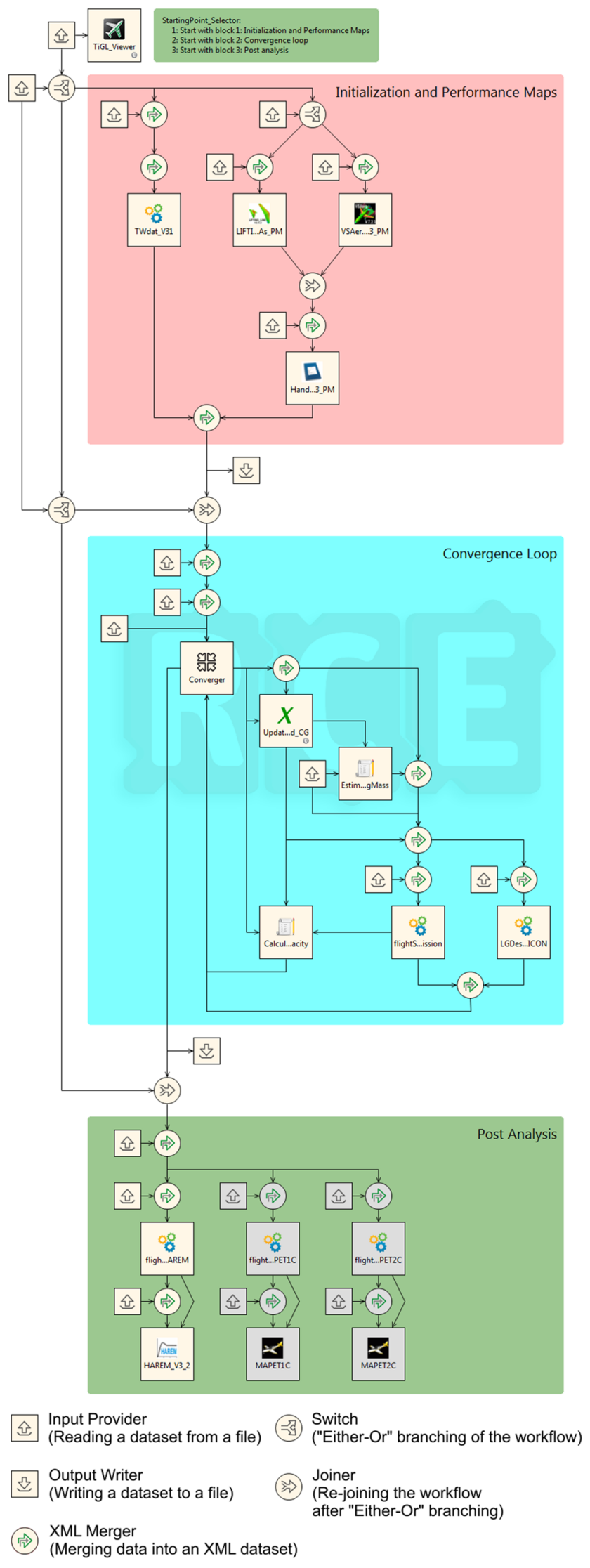

Fig. 5 MULDICON conceptual design workflow a complete CPACS dataset which is passed from one tool to the other-however, it can also be a set of one or more single parameters which is handed over. The small squares are standing either for reading (arrow up), or writing (arrow down) modules. The first ones are reading and providing additional data to the process; the latter ones are saving the current state of the CPACS dataset to a storage medium. The small circles (containing gray arrows) are responsible for branching and re-joining together the workflow, or (gray arrows with green outline, called "XML Merger") to join together data coming from two sources. The combination of a reader module with an XML Merger is typically used to infuse additional data into the dataset. Such a combination can be seen prior to each of the analysis tools, where toolspecific control data for the tools are provided.

The workflow starts with two reader modules in the upper left corner of the diagram. The topmost one reads the initial CPACS dataset and passes it to a TiGL Viewer component for displaying the geometry. The leftmost reader component provides a control parameter, specifying where to start the workflow (red, blue or green step). The other branching and joining components outside the marked steps are responsible for passing the initial CPACS dataset to the beginning of the selected step.

The first step ("Initialization and Performance Maps", marked in red) immediately splits up into two parallel branches. The first (left) one of them creates a performance deck for the engine, containing all relevant engine parameters (e.g. mass flows, temperatures, emissions) over a variety of altitudes, Mach numbers and thrust settings. The DLR propulsion tool "TWdat" used here is a database, fed with a number of engines and providing the opportunity to apply a "rubber scaling" to scale the engine to a demanded static thrust. The engine design itself is performed in advance in a separate gas turbine simulation environment [29]. Aside from the performance map, TWdat also provides a set of global engine parameters like mass and fan diameter.

The second branch (right) creates a set of aerodynamic performance maps: The first map contains force and moment coefficients over a variety of Mach numbers, Reynolds numbers, angles of sideslip, and angles of attack for the "clean" configuration without control surface deflections. On top of this four-dimensional clean configuration dataset, a fivedimensional delta-coefficient performance map is created for each control surface (introducing the deflection of the control surface as fifth dimension). By superposition of different control surface delta-coefficients with the absolute coefficients of the clean configuration dataset, it is possible to combine the deflections of multiple control surfaces. A third set of aerodynamic performance maps contains the 18 damping derivatives ( 6 coefficients $\times$ rotation around the 3 axes) for each point of the clean configuration dataset. Depending on the number of each of the dimensions' entries and on the 
number of control surfaces, this aerodynamic dataset may grow quite large. In fact, for the MULDICON dataset used here, it accounts for a number of 45500 entries in total. Even with modern computer systems, it is not possible to handle such a number of RANS-CFD computations in an acceptable timeframe- but using simple, potential flow theory-based aerodynamics methods, a performance deck with this size can be created within a few hours or even within minutes. In this process chain, it can be selected whether DLR's open source "LIFTING_LINE" method [30-32] shall be used, or Analytical Methods' commercial "VSAERO" tool [33]. In combination with DLR's simple "HandbookAero" method which accounts for viscous drag and wave drag, a rather complete aerodynamic dataset can be created. In these methods, simple control surfaces (without fowler movement) can be modeled just by rotating the normal vectors of the corresponding mesh panels. Control surfaces like spoilers, which cannot be modeled with inviscid, potential flow based methods, can either be applied via HandbookAero using guessed control derivatives from handbook formulas, or they can be taken from higher fidelity methods and integrated here manually. At the end of the red block, the resulting performance maps for propulsion and aerodynamics are merged together and the complete CPACS dataset is written to the selected storage medium.

Thereafter, the workflow directly continues with the second step ("Convergence Loop", marked in blue), which is the convergence loop for aircraft sizing. Typically, this loop would incorporate a mission fuel calculation and a structural sizing process. In the Mephisto project, where the geometry was fixed, the structural sizing process was replaced by applying the structural mass from a separate, higher fidelity structural design work [34-36]. Thus, with respect to structures, only the landing gear sizing remains directly in the convergence loop. The loop starts with three blocks, integrating template data structures for mass breakdown and weight and balance, as well as starting parameters for the convergence loop. The big "Convergence" square is the driver component for an iteration loop in the workflow. It repeats the rest of the blue part until the computations of design mission fuel and landing gear mass have converged. Within the iteration loop, first comes a Microsoft Excel spreadsheet (big square with green " $\mathrm{X}$ ") for calculating mass, CG location, and mass moments of inertia for 11 selected weight and balance cases. Right behind it follows a small script which is responsible for deriving a maximum landing mass for sizing the landing gear. Currently, this is just done by taking a constant factor of 0.9 from the MTOM. Then, the outputs from Converger, Excel spreadsheet and landing mass script are joined together in another XML Merger component before the workflow splits up into two parallel branches. The left one runs the DLR "flightSim" tool, a 3/6 degree of freedom flight simulation tool [37], which is used here in the 3 degree of freedom mode to perform a simulation of the design mission. As a result, the flight trajectory and the required fuel are written back to the CPACS file. In the right branch the DLR tool "LGDesign" is used to analyze and size the landing gear of MULDICON [38]. As a result, it provides mass and geometry of the landing gear, which is combined with the mission fuel mass and trajectory from flightSim and fed back into to Converger module for the next iteration. The "CalculateRequiredFuelCapacity" component (big square in the lower left corner of the blue region) is just a simple script which computes a required fuel capacity in percent based on the mission fuel demand resulting from flightSim. At the end of the blue block, the aircraft is completely sized and the results are again written to a CPACS file.

The third step is called "Post Analysis" and marked in green. It consists of different analysis tools, further investigating the properties of the aircraft. On the left side of the workflow, there is "HAREM", a DLR analysis tool for investigating and evaluating the handling qualities of an aircraft $[11,39,40]$. In this branch, the flightSim tool is used again, but this time, it creates a 6 degree of freedom dynamic aircraft model which is handed over to HAREM. HAREM is used to assess some of the design requirements like the roll performance. The other two branches are intended for future flight performance and landing performance investigations, however, they are not used yet. To enhance handling qualities and aircraft performance (semi-) automatically, this post-analysis step could also be coupled in a convergence loop, parametric study, or geometry optimization process in the future. For MULDICON-like configurations with their vortex-dominated flow fields, this would require a fast, robust, and automatic high-fidelity aerodynamics process which is still not available yet. Thus, in Mephisto the control surface design was still done manually [13, 41, 42].

\section{Aerodynamic design studies}

As described in Sect. 2.2, the need for several aerodynamic enhancements was specified as part of the MULDICON design task. An overall design to achieve an optimal aerodynamic performance for the entire flight envelope would be performed best using an inverse design methodology. However, the classical inverse design methods rely on aerodynamic models capturing the design point but not the nonlinear aerodynamics in the high angle of attack off-design range. Thus, they are not appropriate for geometry design for vortex-dominated flows typically appearing on highly swept wings for medium to high angles of attack. As a solution, the vortex effects could be investigated separately and the results and knowledge gained from that investigation could be joined together with inverse design results of not vortex-dominated flow cases to come to an enhanced overall 


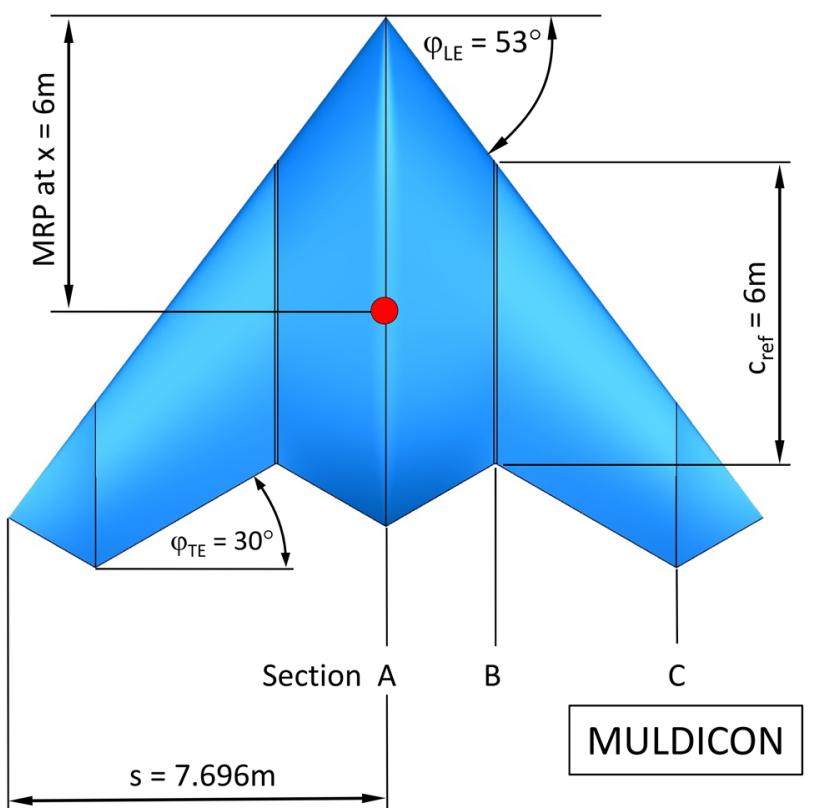

Fig. 6 Planform and reference data of MULDICON

concept. The following sections are dedicated to the first part of the solution sketched above. They present some design studies which were performed to investigate the effects of specific geometric changes on aerodynamic performance as well as on longitudinal stability and control characteristics. A more detailed view on these studies is published by Schütte et al. [43]. The geometric properties of the MULDICON model used here are sketched in Fig. 6.

\subsection{Airfoil studies}

This first section discusses the influence of applying different airfoils at sections A, B and C. The results of the numerical simulations using the DLR solver TAU [44] are shown in Fig. 7. Depicted are the lift and pitching moment coefficient for the four different airfoils. The Baseline configuration uses the SACCON airfoils applied to the MULDICON planform. The other three are a typical Super Critical airfoil, a symmetric NACA-64A-010 and a cambered NACA-65A-410 airfoil. Besides the Baseline, the other airfoils are adapted with a CST [45] approach to apply a constant leading edge curvature radius of $r=1 \mathrm{~mm}$, related to a normalized chord length of $1 \mathrm{~m} .^{8}$

The aim of this investigation is to provide a less complex topology in comparison to the SACCON leading edge contour distribution to get rid of the discontinuity in the

\footnotetext{
$\overline{8}$ This means that the leading edge radius distribution of the aircraft depends on the airfoil scaling due to local chord length.
}
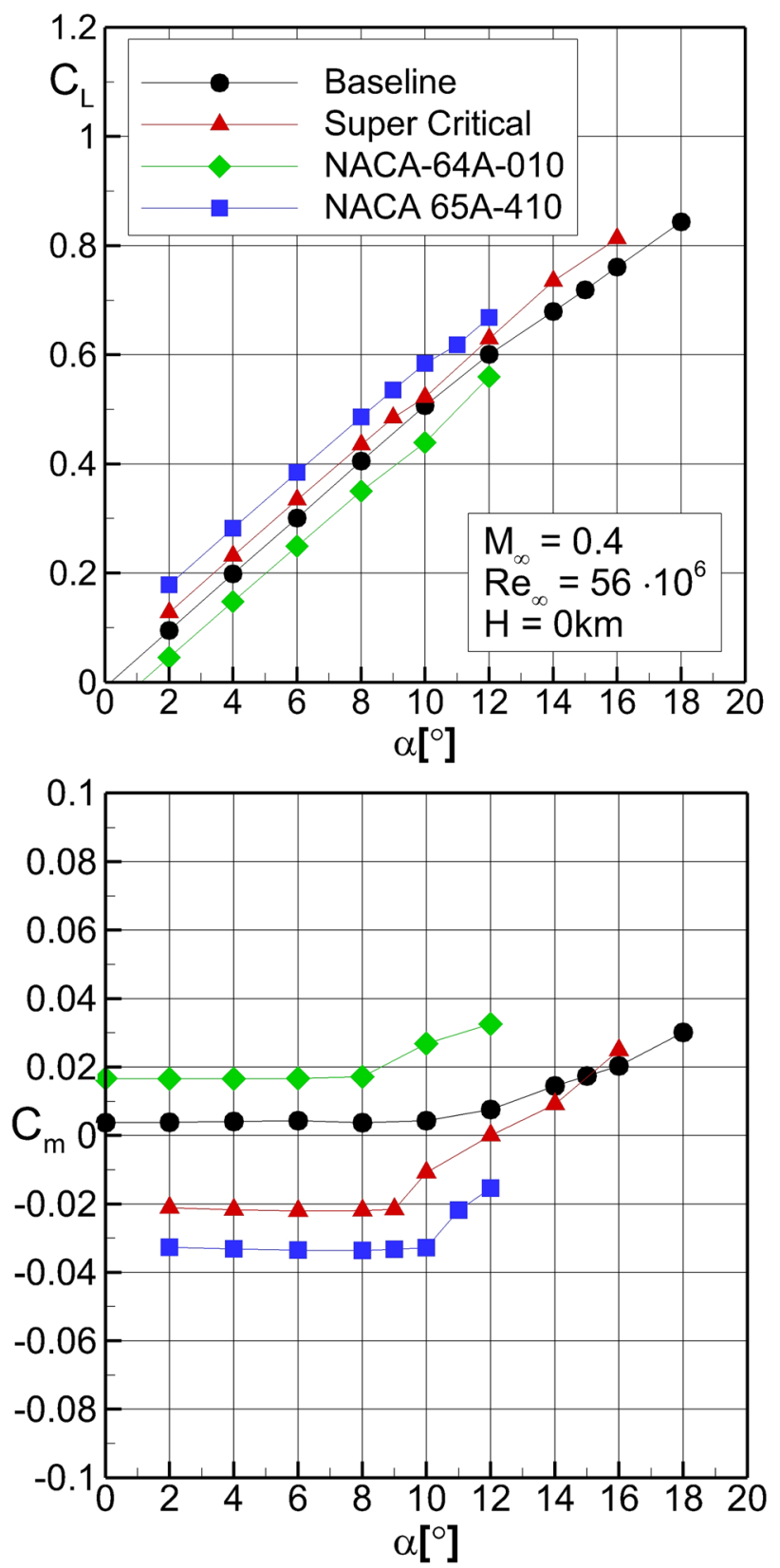

Fig. 7 Lift coefficient (top) and pitching moment coefficient (bottom) versus angle of attack for MULDICON with different airfoils

pitching moment gradient which occurs for SACCON at higher angles of attack, as described amongst others by Schütte et al. [14].

The lift distribution versus angle of attack shows a quite similar linear behavior for all airfoils. The differences between the four sets of airfoils are directly related to their different cambering. The non-cambered NACA-64A-010 airfoil provides the lowest lift coefficient followed by the Baseline configuration, the Super Critical and NACA65A-410 airfoil. The pitching moment coefficients show a 


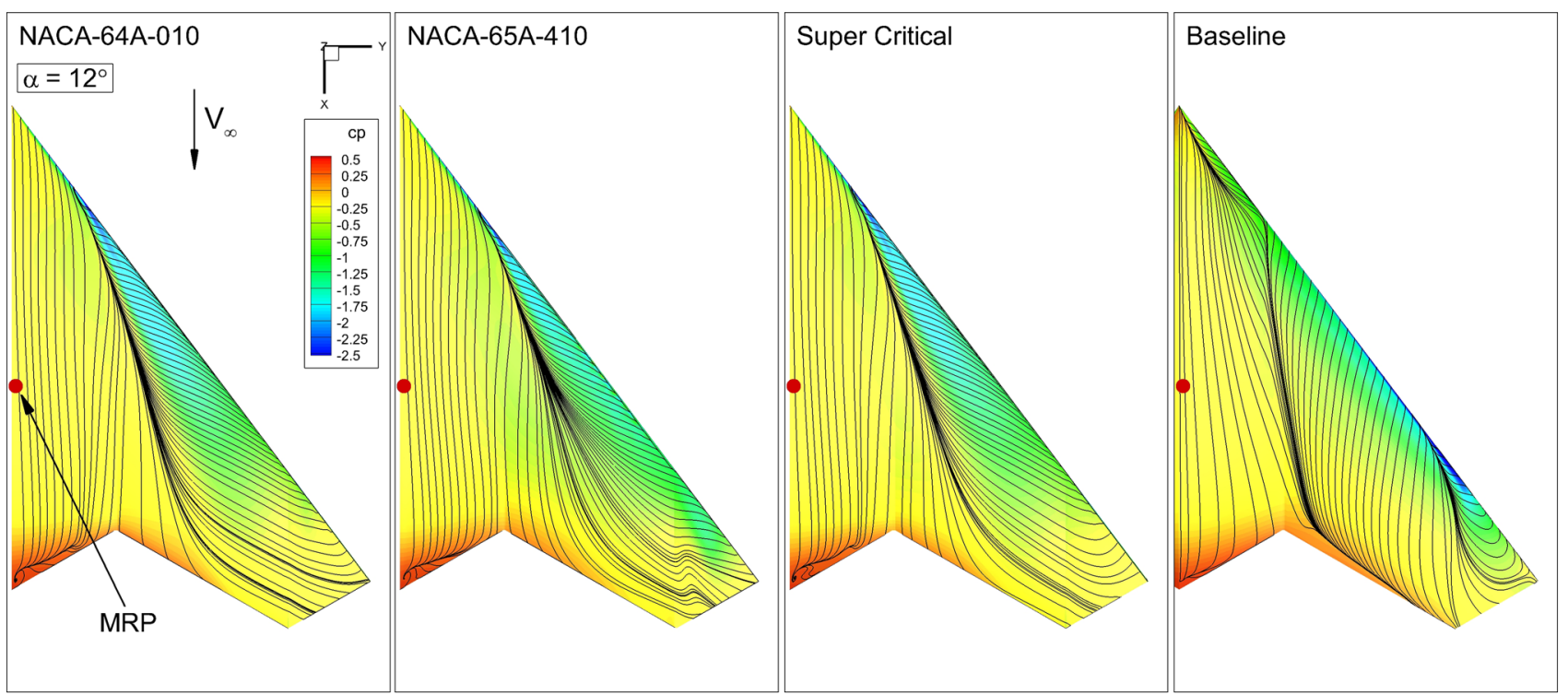

Fig. 8 Pressure distribution and skin friction lines for different airfoil setups $\left(M_{\infty}=0.4, \operatorname{Re}_{\infty}=56 \cdot 10^{6}, \alpha=12^{\circ}\right.$, upper side $)$

neutral stability behavior ( $C_{m} \approx$ const.) for angles of attack from $0^{\circ}$ to $8^{\circ}$ for all applied airfoils. That means that the moment reference point (MRP) is placed in the neutral point. For the Baseline the pitching moment coefficient is around zero, whereas the Super Critical and NACA-64A-410 airfoils provide negative values and the symmetric NACA airfoil positive ones. Beyond $\alpha=10^{\circ}$, the pitching moment gets a positive gradient for all configurations, meaning that the neutral point moves forward.

Figure 8 explains the movement of the neutral point. It shows that for all configurations (except for the Baseline) a strong leading edge vortex has been developed at $\alpha=12^{\circ}$. These leading edge vortices provide a higher suction in front of the MRP in comparison to the suction behind it. For angles higher than $\alpha=12^{\circ}$, this trend does not change, because with increasing angle of attack the starting point of the vortex at the leading edge moves towards the apex. This vortex development for swept wings with round leading edges has been already described by Schütte [46] and should be one of the elements for the further design studies in this article.

The results for the Baseline (SACCON) airfoil configuration are presented to show the effect of the leading edge contour changes from a sharp/round/sharp to a constant round leading edge. The progress in comparison to SAC$\mathrm{CON}$ is that the vortex topology is changed from a double to a simpler single vortex topology. However, more important is to note that the pitching moment characteristic is primarily defined by the leading edge contour since the vortex occurrence, its progression and strength depends on the leading edge contour and not on the geometry of the airfoil.

\subsection{Leading edge contour studies}

Referring to the results from the previous section, the question raises how to influence the flow topology over the wing in a way that the vortex development leads to a stable or at least less unstable pitching moment characteristic with respect to the given moment reference point. This question leads to the second parametric study on the MULDICON planform dealing with the effect of the leading edge radius regarding the vortex development and related effects on the pitching moment characteristic.

Therefore, several constant varying radii cases were simulated. In this case the Super Critical airfoil from the previous section has been applied with a leading edge curvature of $r=1$ (like in Fig. 7), 3, and $5 \mathrm{~mm}$, again related to a normalized chord length of $1 \mathrm{~m}$.

The results in Fig. 9 show that with increasing leading edge radius the vortex occurrence at the leading edge and movement towards the apex will be pushed to higher angles of attack. This confirms the findings from the previous section, that the vortex occurrence and progression is driven by the leading edge shape and independent from the general shape of the airfoil. The values and gradients of lift and pitching moment coefficient below the point where the vortices occur remain almost the same. Beyond that point, even for the biggest leading edge radius, the gradient of the pitching moment coefficient is still positive, thus unstable. As a consequence, further design efforts are required to come to the desired neutral stability. 

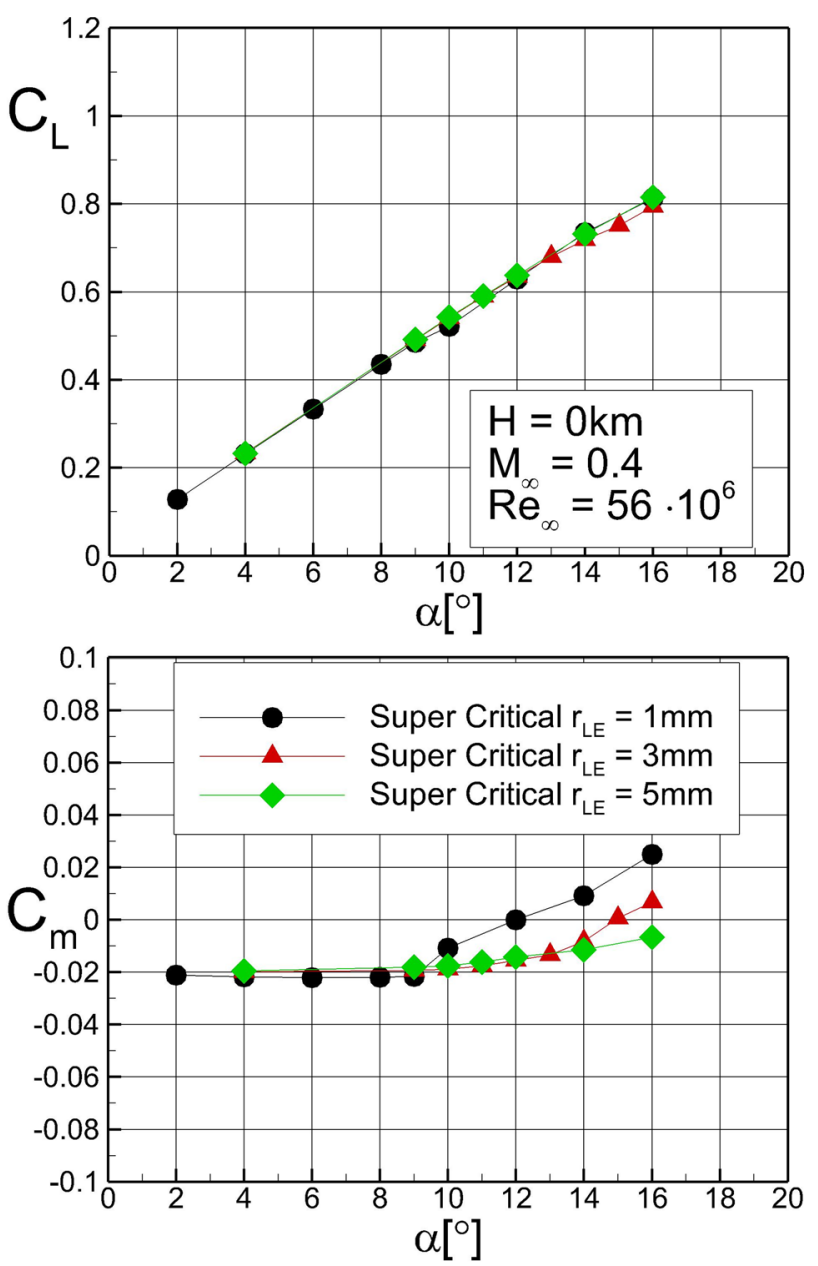

Fig. 9 Lift coefficient (top) and pitching moment coefficient (bottom) for a leading edge contour study applying the DLR RANS solver TAU

\subsection{Airfoil twist and wing sweep studies}

The third study investigates the effect of twist and leading edge sweep angle variations. The intention of this study is to enhance the effect described in the previous section by decreasing the local angles of attack due to a different twist distribution and by changing the wing tip to strengthen the outer vortex.

Three different configurations (Case 7, 8 and 9) based on the NACA-65A-410 airfoil have been applied. For all of them the leading edge radius at section $\mathrm{A}$ and $\mathrm{B}$ is $r=3 \mathrm{~mm}$. The leading edge radius is decreasing then from $r=3$ to $r=0 \mathrm{~mm}$ from section B to section $\mathrm{C}$ and remains sharp towards the outer wing tip. The twist at section $\mathrm{A}$ is $\varepsilon_{\mathrm{TE}}=0^{\circ}$, for section B it is $\varepsilon_{\mathrm{TE}}=-2^{\circ}$, rotated around the trailing edge. This inner wing twist is applied to reduce the effective angle of attack, to keep the vortex further outboard with increasing $\alpha$. The sharp leading edge should increase the strength of the vortex affecting the surface and the load distribution behind the MRP. The difference between Case 7 and 8 is the twist of section $\mathrm{C}$ being $\varepsilon_{\mathrm{LE}}=0^{\circ}$ for Case 7 and $\varepsilon_{\mathrm{LE}}=-3^{\circ}$ for Case 8 , rotated around the leading edge. For Case 9, the leading edge tip sweep angle of Case 8 has been increased from $53^{\circ}$ to $65^{\circ}$. The aim is to strengthen the vortex to increase the load behind the MRP, thus reducing the gradient of the pitching moment. As an overview, the parameters of the three cases are shown in Table 2.

In the same manner as for the previous design studies, the lift is not much affected by the design changes. On the other hand, the pitching moment coefficient changes from case 7 to case 9, as plotted in Fig. 10. Comparing case 7 and 8 it can be seen that the twist change shifts the center of pressure forward, but barely has an effect on the neutral point. For case 9 , the center of pressure is located even more forward, but the expected reduction of the gradient (standing for the desired rearward movement of the neutral point) seems to be very small and occurring at very high angles of attack only.

Figure 11 shows the flow topology of case 8 and 9 for an angle of attack of $20^{\circ}$. It can be seen that both cases look quite similar, as there is no major impact regarding a stronger stable vortex stabilizing the tip vortex. Thus, there is no significant movement of the neutral point.

Concluding these studies, it has been shown that only the variation of the leading edge radius proved to be a useful measure for reducing the discontinuity in the pitching moment gradient, even though this is not sufficient to keep the neutral point fixed for higher angles of attack. That means that to keep the design requirement of having neutral stability for the most rearward CG location, the permitted rearward CG would have to be shifted forward.

An example of how the pitching moment characteristic would change for an MRP (corresponding with the rearward CG) of $5.5 \mathrm{~m}$ instead of $6.0 \mathrm{~m}$ is depicted for case 8 and 9 in Fig. 12. This approach would lead to a stable stability (negative gradient of pitching moment) for angles of attack below $12^{\circ}$. Above $12^{\circ}$ the gradient is slightly positive for Case 8 before it becomes neutral. A similar but even more neutral behavior can be observed for Case 9. However, this
Table 2 Twist and sweep cases (NACA-65A-410)

\begin{tabular}{|c|c|c|c|c|c|c|c|}
\hline \multirow[t]{2}{*}{ Case } & \multicolumn{2}{|c|}{ Section A } & \multicolumn{2}{|c|}{ Section B } & \multicolumn{2}{|c|}{ Section C } & \multirow{2}{*}{$\begin{array}{l}\text { Tip } \\
\varphi_{\mathrm{LE}}\end{array}$} \\
\hline & $r[\mathrm{~mm}]$ & $\varepsilon_{\mathrm{TE}}$ & $r[\mathrm{~mm}]$ & $\varepsilon_{\mathrm{TE}}$ & $r[\mathrm{~mm}]$ & $\varepsilon_{\mathrm{TE}}$ & \\
\hline 7 & 3 & $0^{\circ}$ & 3 & $-2^{\circ}$ & 0 & $0^{\circ}$ & $53^{\circ}$ \\
\hline 8 & 3 & $0^{\circ}$ & 3 & $-2^{\circ}$ & 0 & $-3^{\circ}$ & $53^{\circ}$ \\
\hline 9 & 3 & $0^{\circ}$ & 3 & $-2^{\circ}$ & 0 & $-3^{\circ}$ & $65^{\circ}$ \\
\hline
\end{tabular}




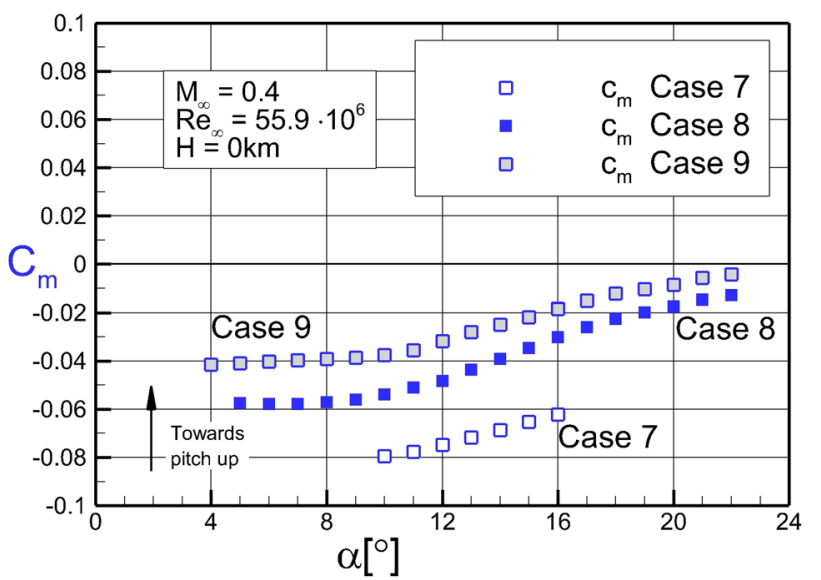

Fig. 10 Pitching moment coefficient versus angle of attack for Case 7,8 and 9

means that there is still a movement of the neutral point of more than $8 \%$ MAC between low and high angles of attack, compared to a desired CG range of only $3 \%$ MAC.

\subsection{Engine integration}

The purpose of this section is to investigate the influence of the aerodynamic performance by integrating an engine intake and outlet and applying the related engine conditions to MULDICON. In Fig. 17 in the appendix, the configuration for case 8 is shown with a generic integrated engine intake and nozzle. It can be seen that the volume of the inner wing body section has not been increased but reduced between the engine inlet duct and the apex. The boundary conditions of the inlet and outlet surfaces as well as the engine conditions have been evaluated within a separate investigation within the AVT-251 group [47].

For the calculations with engine, the "Approach" case from the design requirements has been selected (see Table 5). It consists of a Mach number of 0.4 , a vertical load factor of 2.5 and an aircraft mass of $13000 \mathrm{~kg}$. Figure 13 shows a comparison of the lift and pitching moment coefficient between a configuration with and without integrated engine.

The plot shows that the lift and pitching moment characteristic are quite similar for both cases within the considered angle of attack range. The engine integration is neither affecting the longitudinal stability, nor the overall flow topology on the upper wing surface. Figure 14 shows the lift, drag and thrust coefficient for the configuration with engine integration for angles of attack between $2^{\circ}$ and $10^{\circ}$. In addition to that, the load factor is evaluated. For the selected "Approach" design point, the load factor of 2.5 is provided at $\alpha=6^{\circ}$. A comparison between drag and thrust coefficient shows a slight lack of thrust of $\approx 80$ drag counts (0.008). This is caused by the installation effects in comparison to the isolated engine evaluation and needs to be adjusted to receive a trimmed flight condition. Nevertheless, in this case the present design matches the requirements quite well.

Finally, Fig. 18 in the appendix shows a comparison of the surface pressure distribution and flow topology on the upper surface at $\alpha=10^{\circ}$. It visualizes that the flow topology is not changed for the current flight conditions with and without engine integration. Furthermore, it can be seen that
Fig. 11 Surface pressure and vortex topology on the upper side of the wing for case 8 and 9 at $\alpha=20^{\circ}$
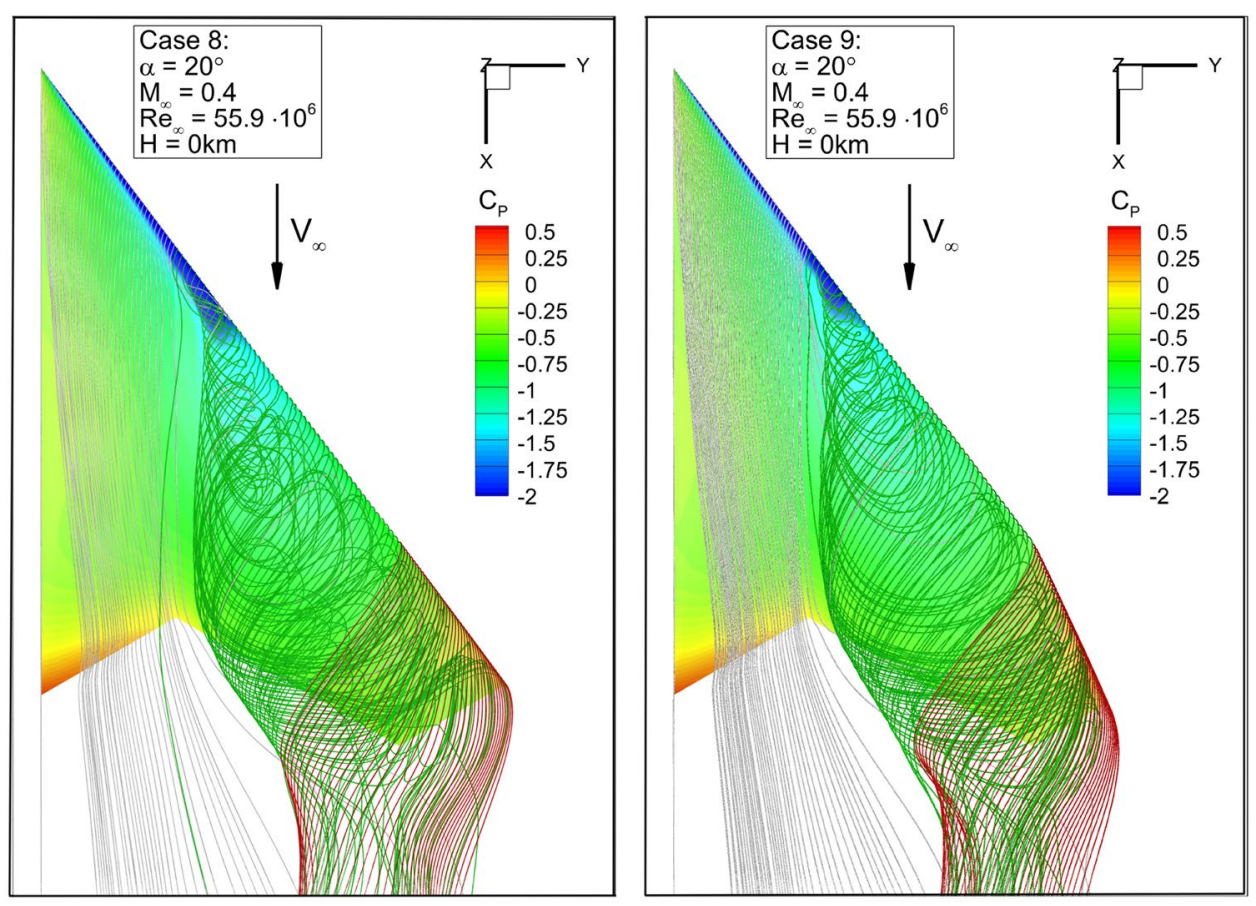


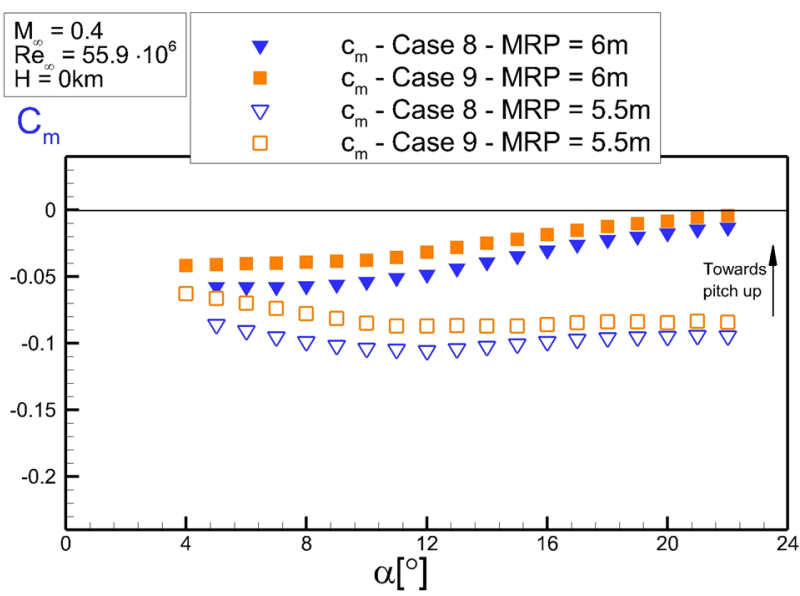

Fig. 12 Pitching moment characteristics for Case 8 and 9 for two different MRP locations

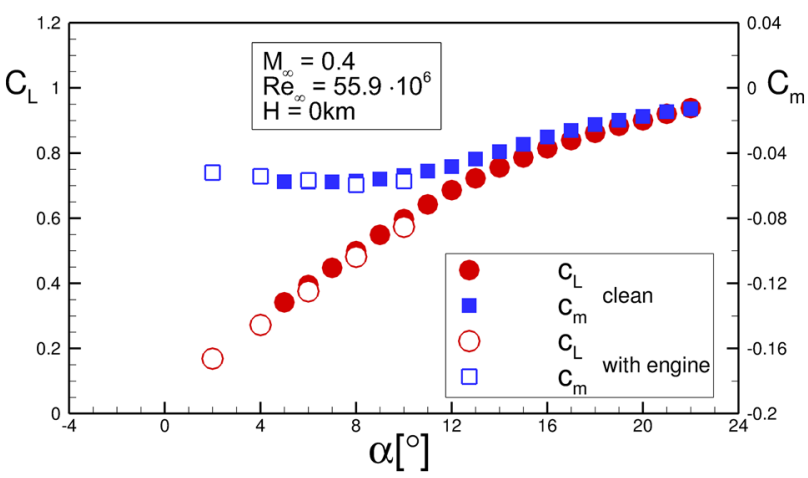

Fig. 13 Lift and pitching moment versus angle of attack for case 8 with and without engine integration

\section{MULDICON design}

As mentioned before, the design of the MULDICON configuration was a collaborative effort between the Mephisto project and AVT-251. The first MULDICON geometry was a reference configuration, which is identical to SACCON (same airfoils, same twist distribution), but with the modified trailing edge sweep of $30^{\circ}$. To satisfy the requirements for maximum lift coefficient and pitching moment characteristics, two different design philosophies were applied to the reference configuration. The first one was focused on the understanding of the physical principles behind the complex vortex phenomena. This one was part of the DLR Mephisto project and is described in Chapter 4 of this article. As its best result case 8 was taken. The second design approach was performed within AVT-251. In contrast to the DLR work, it aimed at minimizing vortex effects by designing for attached flow conditions. Therefore, a complete redesign of airfoil shapes and twist distribution was performed. Finally, the discontinuities in the pitching moment could be reduced and the maximum lift coefficient was increased. Details about this second, inverse design approach are given by Nangia et al. [48]; Table 3 shows its achievements with respect to maximum lift coefficient. It can be seen that the design targets for these three points are nearly met. For the "Combat High Altitude" case, the maximum lift coefficient is slightly lower than the design target, however, the lift demand for the $4.5 \mathrm{~g}$ manoeuver is still reached.

For the conceptual design studies, all three versions (reference configuration and the two new designs) were modeled in CPACS and investigated in the conceptual design workflow. At this point it turned out that the DLR design case 8

Fig. 14 Lift, drag, thrust and load factor versus angle of attack for case 8 with integrated engine

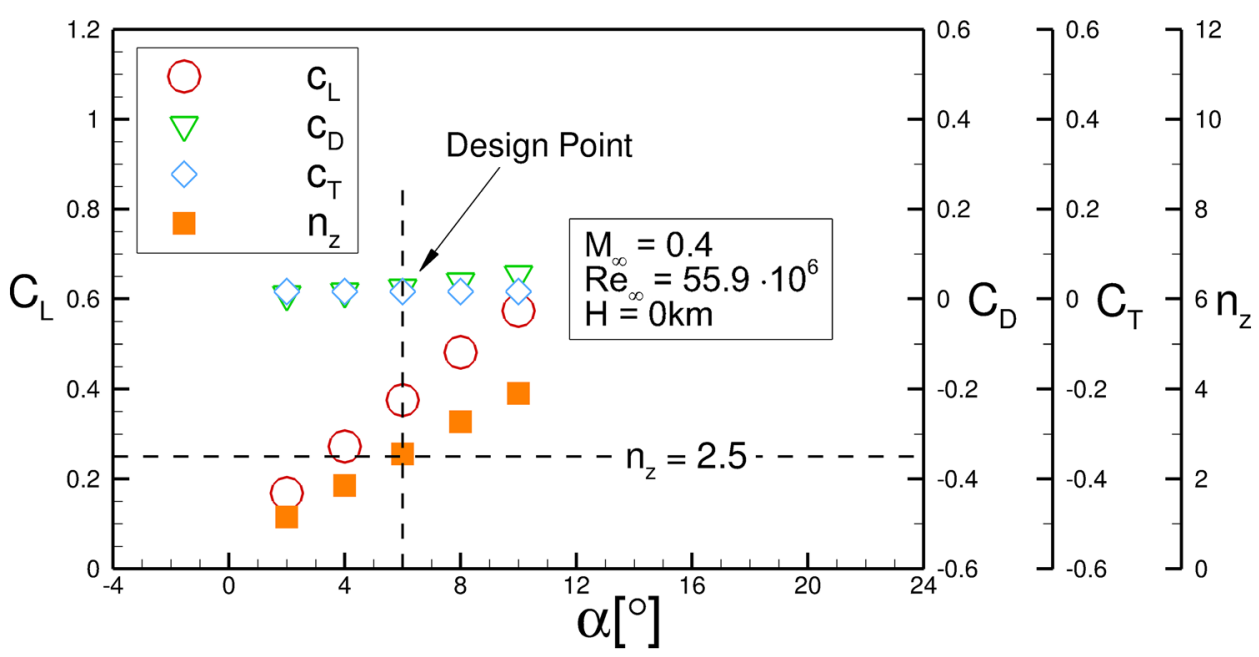

the adjusted apex geometry for the configuration with engine inlet is not causing a flow topology which could harm the required regular onflow condition for the engine inlet. is not yet usable as its generic airfoils incorporate too much camber (causing a strong zero-lift pitch-down moment) for a 
Table 3 Maximum lift coefficients of MULDICON compared to requirements

\begin{tabular}{lll}
\hline Flight case & Takeoff & $\begin{array}{l}\text { Combat } \\
\text { High Alti- } \\
\text { tude }\end{array}$ \\
\hline Vertical load factor & 1.5 & 4.5 \\
Required lift coefficient & 1.0 & 0.717 \\
Target value for maximum lift coefficient & 1.1 & 0.817 \\
MULDICON baseline (SACCON airfoils) & $0.84-0.96$ & 0.61 \\
MULDICON final design & $1.11-1.14$ & 0.72 \\
\hline
\end{tabular}

flying wing aircraft. Since there were no resources available to apply the leading edge design to a more suitable set of airfoils, this concept was not investigated further. Thus, the second design concept, which is the official final design from AVT-251 is taken as main concept for the conceptual design studies (see Fig. 19 in the appendix).The Aerodynamic shaping work within AVT-251 as a whole is documented by van Rooij and Cummings [49].

The second main task in the development of MULDICON was the design of a suitable control concept, which is discussed by Löchert et al. [13, 42]. After confirming that the conventional trailing edge devices for roll and pitch worked much better than they did for SACCON, the focus of this work was placed upon finding a solution for yaw control. As it was not clear at this point, how much yawing moment coefficient would be required, a target maximum value of 0.015 was chosen based on experience. To validate this value, an investigation with varying yaw control efficiency was performed by Hasan et al. [50] (Fig. 25, p. 27). Using the conceptual design workflow for simulating a landing maneuver with maximum permitted crosswind, it turned out that a yawing moment requirement of 0.015 seems to be reasonable for handling the permitted crosswind of 30 knots. To reach this yawing moment coefficient without generating too much rolling and pitching moments and without having too much dependency on the angle of attack, a pair of split flaps at the wingtips seemed to be the most promising solution. The final control concept with conventional trailing edge devices for roll and pitch and split flaps for yaw control was applied to the MULDICON CPACS datasets.

Another important task was to provide an engine model which satisfies the engine design requirements, as specified in Sect. 2.2.5. This work was part of the Mephisto project and provided to AVT-251. Starting from a permitted fan diameter of $1 \mathrm{~m}$, some engine design studies were performed. As it became clear that the fan diameter is still critical with respect to the integration of the engine and a corresponding intake and nozzle concept, a variation study for the fan diameter was performed. As a final result, a slightly smaller engine was selected and its performance tables were provided for MULDICON design. The engine design
Table 4 Parameters of MULDICON engine "UCAV_G"

\begin{tabular}{llll}
\hline Parameter & Condition & Unit & Value \\
\hline Static thrust (dry) & Takeoff & $\mathrm{kN}$ & 60 \\
Bypass ratio & Cruise & - & 1.7 \\
Overall pressure ratio & Takeoff & - & 30.5 \\
Mass flow & Takeoff & $\mathrm{kg} / \mathrm{s}$ & 114 \\
Turbine entry temperature & Takeoff & $\mathrm{K}$ & 1740 \\
Specific fuel consumption & Cruise & $\mathrm{g} /(\mathrm{kNs})$ & 23.8 \\
Fan diameter & All & $\mathrm{m}$ & 0.908 \\
Length & All & $\mathrm{m}$ & 2.2 \\
Mass & All & $\mathrm{kg}$ & 1040 \\
\hline
\end{tabular}

work and the sizing study are explained by Zenkner and Becker [47, 51]; the main engine parameters are provided in Table 4. With respect to engine integration into MULDICON, several studies were performed within Mephisto and AVT-251. Due to limitations in time and resources, their final results could not be incorporated directly into the overall aircraft concept; however, their demands were considered as boundary conditions where possible. More Details on engine integration work for MULDICON can be found in [51-54].

As the other main components, the structural concept of MULDICON was defined by a joint activity between Mephisto and AVT-251. Based on experience and Finite Element analyses, the main structural elements were placed and sized, and an estimate for the structural mass of MULDICON was given. A special focus had to be placed on the big cutouts due to engine and payload/weapon bays and on aeroelastic effects like body-freedom-flutter. Further details about the structural and aeroelastic design work for MULDICON are presented in [55-58].

Based on the results coming from the different disciplines, the overall aircraft design work at DLR was performed. One of the central elements of the workflow described above is a spreadsheet containing the main components of the aircraft and a two-dimensional planform view including the CG limits (see Fig. 20 in the appendix).

Using this spreadsheet, the main internal components were arranged. Large components e.g. engine were placed directly, as can be seen in the diagram, while smaller components e.g. avionics boxes, for which the geometric properties are not known at this stage of the design cycle, were placed in free areas, assuming that they will have sufficient space there. The filled circles within the components represent the CG position of that component. It was a difficult, iterative procedure to arrange all the components such that they have sufficient space, while the CG positions for all 11 weight and balance cases under investigation were kept within the specified limits between the red dashed lines. The CG range from the center of the aircraft is further displayed in a 
magnified detail sketch on the right side. The most forward location corresponds to the operational empty mass (OEM) (no payload, no fuel) with landing gear up (GU), while the most rearward one represents the ferry flight case (no payload, maximum fuel) with landing gear down (GD). As an impression of the very tight limitations here, it shall be mentioned that for the operational empty mass case (yellow and green squares), the CG movement due to retracting the landing gear already uses around one third of the permitted CG range. A mass breakdown of MULDICON, as calculated with the spreadsheet, is provided in Table 6 in the appendix. It contains the masses of the main components, their center of gravity locations and the mass moments of inertia for the main axis' around $(0,0,0)$. The deviation moments are currently neglected, as well as the center of gravity locations in Z-direction (set to zero). Table 7 in the appendix lists the selected weight and balance cases for MULDICON, together with the mass moments of inertia for the main axis' around the corresponding CG.

One drawback of the spreadsheet is that it only contains a 2D model of the inner geometry, whereas the thickness of MULDICON varies continuously over the chord. As a consequence, it is not possible to determine from this model sufficiently, whether a component really fits into the outer shape. As a solution to this problem, the spreadsheet was extended by a so-called "design table" for Dassault's CATIA CAD software [59]. Combined with an existing CAD model of the MULDICON outer shape which also incorporates intake, nozzle and the control surfaces of the final control concept, the CATIA software uses the construction table to generate the inner components as specified in the spreadsheet. The CATIA 3D model of the UCAV configuration with its main components is shown in Fig. 21 in the appendix.

In the center of the conceptual design process chain, the simulation of the design mission using the DLR flightSim tool is located. After reaching convergence, the results for MULDICON final design show a required fuel mass of $5341 \mathrm{~kg}$ and a flight duration of $3 \mathrm{~h}$ and $18 \mathrm{~min} .{ }^{9}$ Compared to the maximum fuel capacity of $6374 \mathrm{~kg}$, the fuel reserve is $1033 \mathrm{~kg}$ (or $16.2 \%$ of maximum fuel). With respect to an averaged mission fuel burn of $0.45 \mathrm{~kg} / \mathrm{s}$, this reserve would last for another $38 \mathrm{~min}$ of flight; considering a fuel flow of around $0.17 \mathrm{~kg} / \mathrm{s}$, as it is present at the end of the cruise segment right before the final descent for landing, even a duration of $1 \mathrm{~h}$ and $41 \mathrm{~min}$ is achieved. So, the design requirement of providing $\approx 45 \mathrm{~min}$ of fuel reserve after flying the

\footnotetext{
${ }^{9}$ It hast to be mentioned here that the mission simulation currently neglects the trim drag. However, with respect to the very small stability margin and the low zero-lift moment, the trim drag over the mission flight is assumed to be rather low.
}

design mission is satisfied. Compared to the MULDICON Baseline configuration (MULDICON planform with SACCON airfoils), the final design of MULDICON with its optimized airfoil and twist distribution requires $304 \mathrm{~kg}(\approx 5.4 \%)$ less fuel for the design mission. In Fig. 22 in the appendix, the main parameters of the aircraft are plotted over the flight time.

After the end of the conceptual design workflow, a rather comprehensive CPACS dataset of the MULDICON configuration was made available, permitting further, more detailed investigations of the aircraft concept. One such investigation, which has not been published yet, was dedicated to flight performance and flying qualities evaluations. It shows that with respect to roll performance, the requirements for the "Takeoff" and "Combat High Altitude" cases could not be met sufficiently (by deflecting just the outer control surfaces as ailerons), while the other cases are within the specified limits. Furthermore, it turns out that the available thrust for the "Combat Low Altitude" case is not sufficient with respect to the sustained turn requirement-a consequence of a lift-to-drag assumption which turned out to be too optimistic for that case.

\section{Industrial perspective}

"Connected - smart - integrated" are the key words characterizing the main drivers for industry technology initiatives for years to come. Cyberphysic systems or Industry 4.0 initiatives especially drive the design and manufacturing improvement projects within the technology domain "Design \& Digital Factory". Figure of merit is the improvement of aircraft development times by a factor of 2 . To achieve this, product and industrial system co-design driven by digitalization (strong integration of virtualization, modeling, simulation and multi-criteria optimization) is identified as a promising solution. Joint initiatives with DLR like Mephisto around the CPACS/RCE design environment are used to test new ways of working allowing seamless exchange and update of design information and knowledge among all involved stakeholders. Traditionally the design process at industry is Center of Competence $(\mathrm{CoC})$ driven and the work products are developed locally in the centers and then handed over to the next stakeholder in the design process. With more and more complex and integrated systems, this handover process becomes a blocking point when it comes to improvement of development times and especially the manual formatting and adopting of input and output files is error prone. The tests of CPACS, which were conducted during the Mephisto cooperation, proved that using an automated XML exchange format helped significantly to improve data exchange quality between tools and stakeholders. One must not neglect that preparing available tools to 


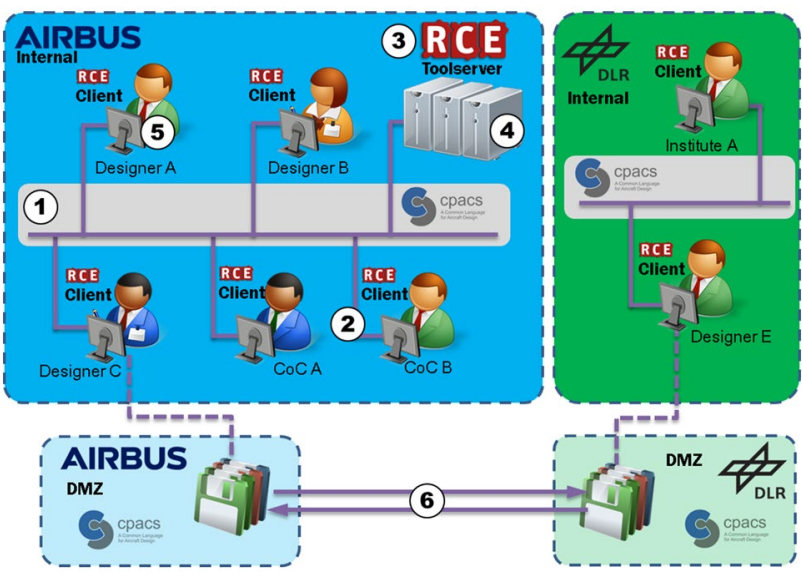

Fig. 15 Setup of design environment and interaction between DLR and AIRBUS

communicate with CPACS and RCE requires a significant effort, but once that hurdle is overcome, iteration times are reduced significantly, error rates are reduced, and the possibility to also integrate automated optimizers into the process can further improve the result quality. Due to security constraints, the network-based approach from DLR is currently not used to connect servers from DLR and AIRBUS directly, but using CPACS as common data exchange format helped to exchange datasets and to work together on the same data.

Figure 15 shows the setup of RCE Network at AIRBUS to simplify the data exchange between future projects office and centers of competence and with DLR. The bullets in the list below describe the interfaces in the figure.

1. Common Data Exchange format within Airbus Conceptual Design (CPACS).

2. Data Exchange format between Airbus Concept Design and $\mathrm{CoC}$ (CPACS).

3. Process definition in RCE framework.

4. Infrastructure for centralized concept design tool server with RCE.

5. Applicability/Feasibility of TiXI/TiGL/Descartes for Airbus Concept Design.

6. Common Data Exchange format for parametric aircraft concept design with external partners.

Nevertheless, the validation of the current CPACS release revealed some gaps in the standard when it comes to describing necessary parameters, especially of defence projects, that need to be closed in future releases to make it fully useable in an industrial environment.

\section{Conclusion and outlook}

Concluding the work from the DLR research project Mephisto which is presented in this article, it can be stated that the MULDICON design task could be fulfilled rather successfully. In close collaboration of the involved disciplines a set of design requirements was specified; with respect to the available resources, the requirements were focused on the most critical aspects. To bring together the disciplinary results, a specific analysis workflow for MULDICON was created using the DLR conceptual design system.

Reviewing the results, it first has to be stated that the mission and payload requirements could be met. Regarding the agility requirements for the specified design points it can be summarized that with the available resources, not all five design points could be investigated to the necessary extent and that the shape design path selected in Mephisto seems promising, but could not be followed up to its end either. However, for the addressed points, the requirements could be satisfied or at least be nearly satisfied. The pitching moment characteristics for both MULDICON design paths have been smoothed-at least for the required range of lift coefficients. The new control concept fulfills most of its requirements, even though the roll performance for the "Takeoff" and "Combat High Altitude" cases is still insufficient; just as the thrust for doing a sustained $4.5 \mathrm{~g}$ turn under "Combat Low Altitude" conditions. With respect to this, coupling the results from the assessment back to disciplinary design and performing another design iteration would have been useful to fulfill the requirements completely. ${ }^{10}$ With regard to structures and aeroelasticity, a suitable solution for the structural concept has been found and investigated.

From a conceptual aircraft design perspective, it has to be noted that a fast and automatic generation of a consecutive aerodynamic database is an essential prerequisite for investigating flight mechanical aspects. The classical low-fidelity aerodynamic methods are able to generate such a database automatically and within a short amount of time, but they are not able to predict the complex aerodynamic effects occurring around highly swept fighter aircraft sufficiently. Such effects can be predicted by modern CFD methods very well, but these methods are still not able to deliver the consecutive database mentioned above automatically and within a short amount of time. A solution to this contradiction can be found in the application of multi-fidelity methods, where the general trends from many low-fidelity data points are combined with the accuracy of selected high-fidelity calculations into a common response

\footnotetext{
${ }^{10}$ Inner and outer control surfaces could be combined to enhance the roll performance, while an additional set of body flaps (to be located left and right to the engine nozzle) could take over the pitch control functionality. Furthermore, an engine redesign loop could be performed to satisfy the missed sustained turn requirement.
} 
surface model. An exemplary application of such a multi-fidelity process on the MULDICON Baseline configuration was already performed within Mephisto and is briefly presented in [50] (Section B.4, pp. 11-14). In the future, this sort of multifidelity aerodynamic datasets can be of great use to investigate the flight mechanic characteristics of agile fighter aircraft concepts already in early stages of aircraft design.

Acknowledgements Open Access funding provided by Projekt DEAL. The authors would like to thank the German MoD and the Federal Office of Bundeswehr Equipment, Information Technology and InService Support (BAAINBw) for their support of the military research presented here. Furthermore, the good cooperation between the DLR research project Mephisto and the NATO STO task group AVT-251 is greatly appreciated.

Open Access This article is licensed under a Creative Commons Attribution 4.0 International License, which permits use, sharing, adaptation, distribution and reproduction in any medium or format, as long as you give appropriate credit to the original author(s) and the source, provide a link to the Creative Commons licence, and indicate if changes were made. The images or other third party material in this article are included in the article's Creative Commons licence, unless indicated otherwise in a credit line to the material. If material is not included in the article's Creative Commons licence and your intended use is not permitted by statutory regulation or exceeds the permitted use, you will need to obtain permission directly from the copyright holder. To view a copy of this licence, visit http://creativecommons .org/licenses/by/4.0/.

\section{Appendix}

See Fig. 16 and Table 5.

See Figs. 17, 18, 19, 20; Tables 6, 7

See Figs. 21, 22.

Fig. 16 MULDICON design mission

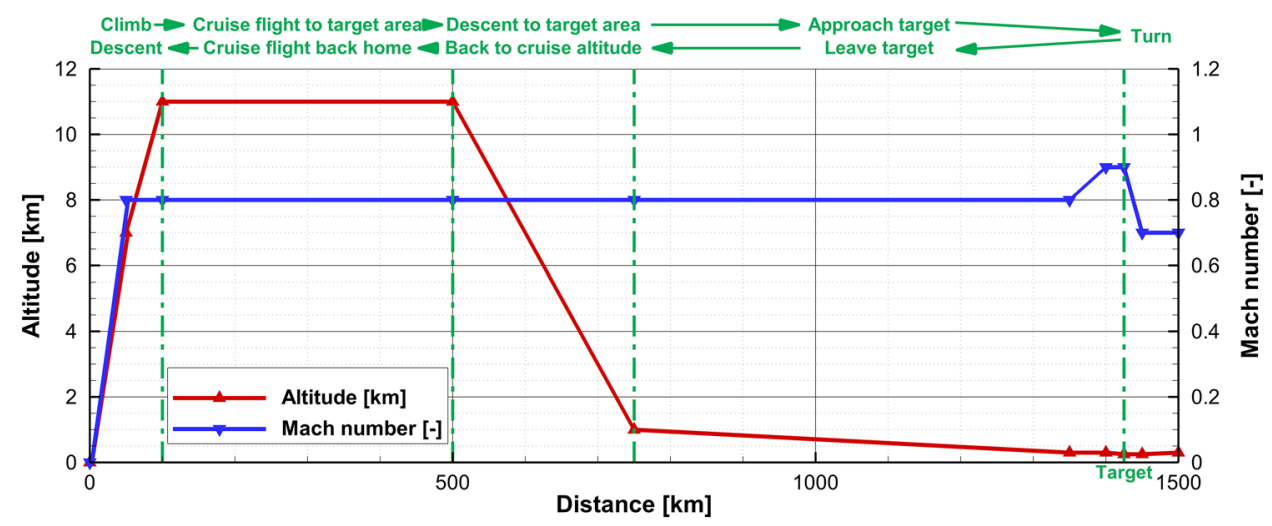

Table 5 MULDICON design points

\begin{tabular}{|c|c|c|c|c|c|c|}
\hline & & Cruise & Takeoff & Combat LowAltitude & Approach & Combat High Altitude \\
\hline \multirow[t]{7}{*}{ Flight conditions } & Altitude & $11000 \mathrm{~m}$ & $0 \mathrm{~m}$ & $0 \mathrm{~m}$ & $0 \mathrm{~m}$ & $11000 \mathrm{~m}$ \\
\hline & Mach number & 0.8 & 0.2 & 0.8 & 0.4 & 0.8 \\
\hline & Velocity & $236 \mathrm{~m} / \mathrm{s}$ & $68 \mathrm{~m} / \mathrm{s}$ & $272 \mathrm{~m} / \mathrm{s}$ & $136 \mathrm{~m} / \mathrm{s}$ & $236 \mathrm{~m} / \mathrm{s}$ \\
\hline & Mass & $15000 \mathrm{~kg}$ & $15000 \mathrm{~kg}$ & $13000 \mathrm{~kg}$ & $13000 \mathrm{~kg}$ & $13000 \mathrm{~kg}$ \\
\hline & Vertical load factor & 1.0 & 1.5 & 4.5 & 2.5 & 4.5 \\
\hline & Approximated turn radius & - & $422 \mathrm{~m}$ & $1722 \mathrm{~m}$ & $824 \mathrm{~m}$ & $1314 \mathrm{~m}$ \\
\hline & Assumed lift-to-drag ratio & 20.0 & 7.0 & 20.0 & 16.1 & 7.0 \\
\hline \multirow[t]{9}{*}{ Design requirements } & Roll performance & $90^{\circ} / 1.7 \mathrm{~s}$ & $30^{\circ} / 1.1 \mathrm{~s}$ & $130^{\circ} / 1.0 \mathrm{~s}$ & $90 \% 1.7 \mathrm{~s}$ & $130^{\circ} / 1.0 \mathrm{~s}$ \\
\hline & Pitch rate & $20 \% \mathrm{~s}$ & $20 \% \mathrm{~s}$ & $20 \% \mathrm{~s}$ & $20 \% \mathrm{~s}$ & $20 \% \mathrm{~s}$ \\
\hline & Yaw rate & $10-15 \% \mathrm{~s}$ & $10-15 \% \mathrm{~s}$ & $10-15 \% \mathrm{~s}$ & $10-15 \% \mathrm{~s}$ & $10-15^{\circ} / \mathrm{s}$ \\
\hline & Permitted crosswind & - & 30 knots $(15.43 \mathrm{~m} / \mathrm{s})$ & - & - & - \\
\hline & Lift coefficient & 0.184 & 1 & 0.162 & 0.361 & 0.717 \\
\hline & Maximum lift coefficient & 0.284 & 1.1 & 0.262 & 0.461 & 0.817 \\
\hline & $\begin{array}{l}\text { (Max. lift coefficient SACCON) } \\
\text { (corresponding angle of attack) }\end{array}$ & $\begin{array}{l}(\approx 0.65) \\
\left(\approx 12-14^{\circ}\right)\end{array}$ & $\begin{array}{l}(\approx 0.90) \\
\left(\approx 20-21^{\circ}\right)\end{array}$ & $\begin{array}{l}(\approx 0.65) \\
\left(\approx 12-14^{\circ}\right)\end{array}$ & $(-)$ & $\begin{array}{l}(\approx 0.65) \\
\left(\approx 12-14^{\circ}\right)\end{array}$ \\
\hline & $\begin{array}{l}\text { Assumed drag coefficient } \\
\text { (for Engine design) }\end{array}$ & 0.0092 & 0.1429 & 0.0081 & 0.0224 & - \\
\hline & $\begin{array}{l}\text { Sustained turn thrust demand } \\
\text { (for Engine design) }\end{array}$ & - & $32.15 \mathrm{kN}$ & $29.23 \mathrm{kN}$ & $20.13 \mathrm{kN}$ & - \\
\hline
\end{tabular}


Fig. 17 MULDICON design case 8 with integrated engine inlet and outlet

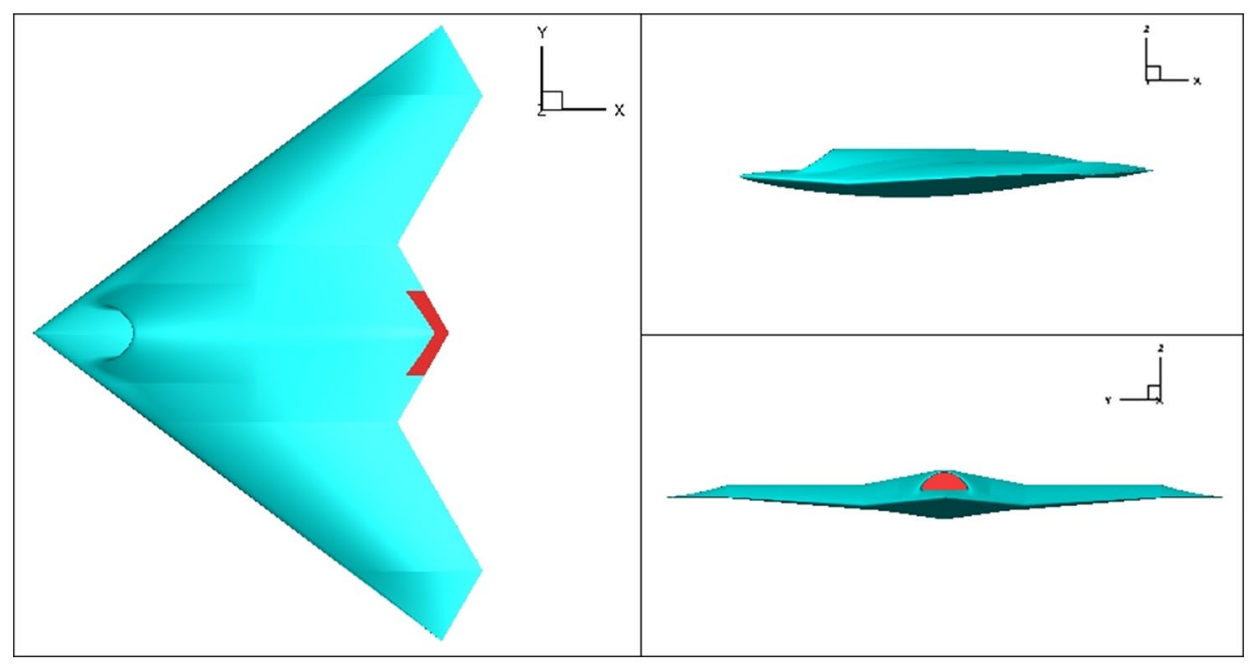

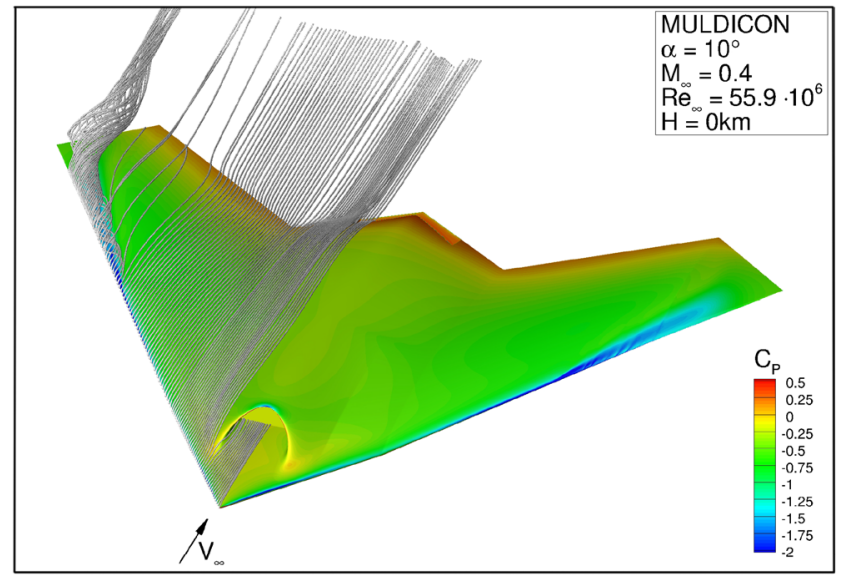

(2.9.10

$\mathrm{Re}_{\mathrm{e}}=55.9 \cdot 10^{6}$

$\mathrm{H}=0 \mathrm{~km}$

1
$\mathrm{C}_{\mathrm{P}}$
$\mathrm{C}_{0.5}$
0.25
0.25
-0.25
-0.5
-0.75
-1.25
-1.5
-1.75
-2.2

Fig. 18 Flow topology on the upper wing of MULDICON design case 8 without (left) and with (right) engine integration
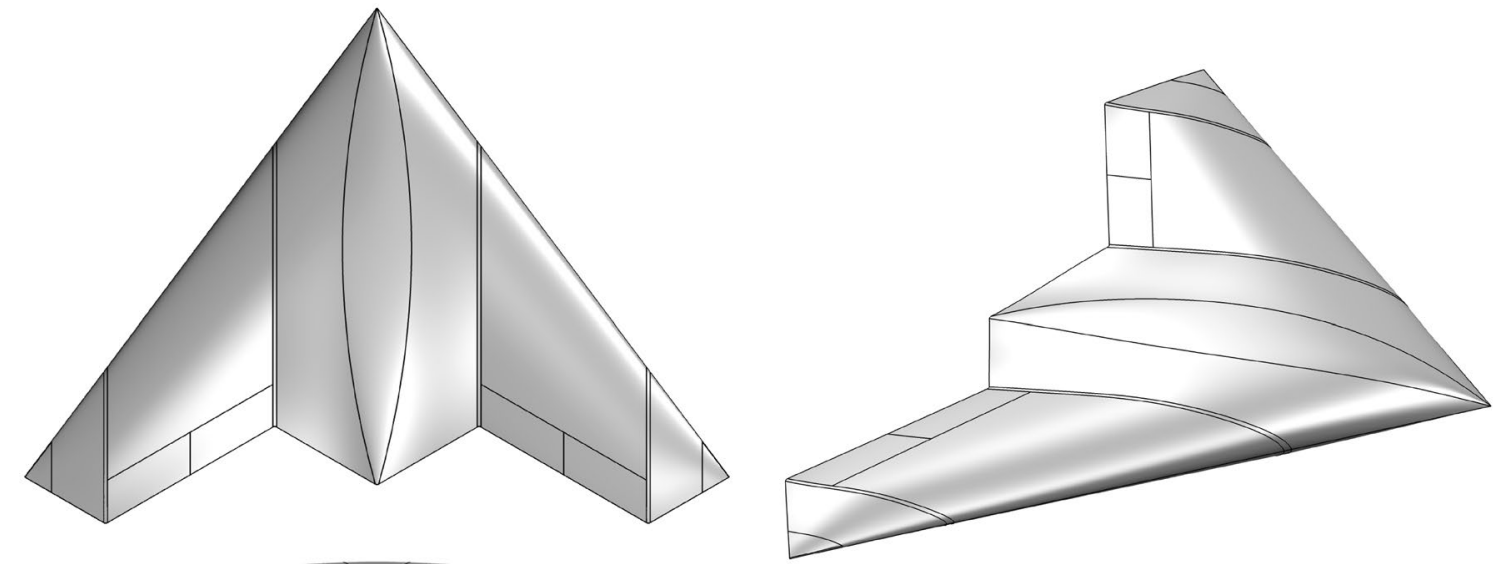

Fig. 19 Outer shape of the MULDICON final configuration 


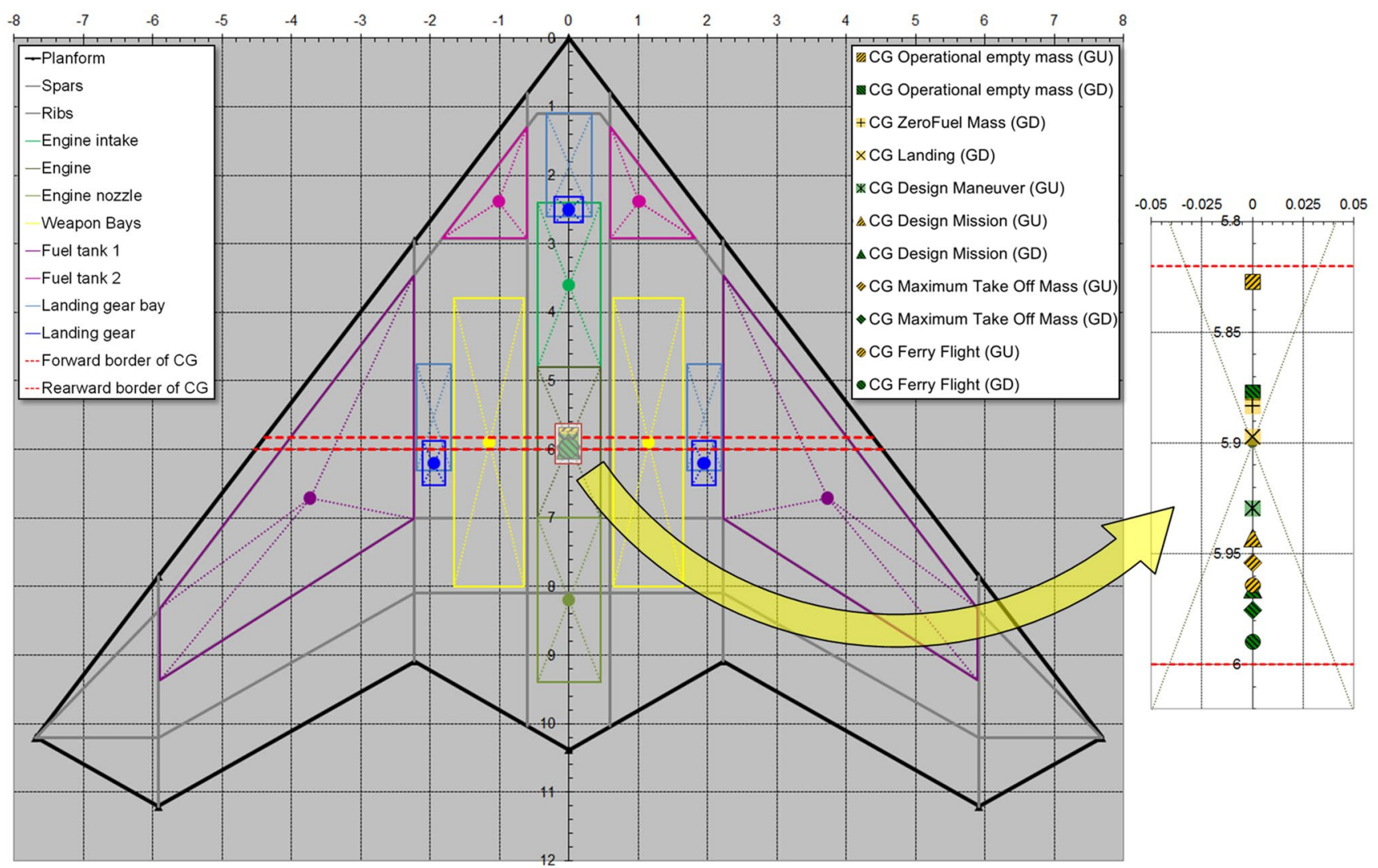

Fig. 20 MULDICON planform with inner arrangement and CG locations

Table 6 MULDICON

component masses, CG locations, and mass moments of inertia around $(0,0,0)$

\begin{tabular}{|c|c|c|c|c|c|c|c|}
\hline \multirow[t]{2}{*}{ Component } & \multirow[t]{2}{*}{ Mass $[\mathrm{kg}]$} & \multicolumn{3}{|c|}{ CG Coordinates } & \multicolumn{3}{|c|}{ Mass Moments of Inertia $(0,0,0)$} \\
\hline & & $X[\mathrm{~m}]$ & $Y[\mathrm{~m}]$ & $Z[\mathrm{~m}]$ & $I_{x x}\left[\mathrm{kgm}^{2}\right]$ & $I_{y y}\left[\mathrm{kgm}^{2}\right]$ & $I_{z z}\left[\mathrm{kgm}^{2}\right]$ \\
\hline Structures & 2638 & 6.84 & 0.00 & 0.00 & 25914 & 124619 & 150533 \\
\hline Landing gear (down) & 321 & 5.29 & 0.00 & 0.00 & 920 & 9796 & 10716 \\
\hline Landing gear (up) & 321 & 4.24 & 0.00 & 0.00 & 920 & 6561 & 7481 \\
\hline Propulsion & 1459 & 5.95 & 0.00 & 0.07 & 10 & 51644 & 51633 \\
\hline Systems & 1790 & 4.49 & 0.00 & 0.00 & 0 & 48939 & 48939 \\
\hline Other & 559 & 5.93 & 0.00 & 0.00 & 595 & 19673 & 20268 \\
\hline OEM (GD) & 6767 & 5.88 & 0.00 & 0.02 & 27440 & 254672 & 282091 \\
\hline OEM (GU) & 6767 & 5.83 & $\mathbf{0 . 0 0}$ & 0.02 & 27440 & 251437 & 278856 \\
\hline Maximum payload & 2500 & 5.90 & 0.00 & 0.00 & 3306 & 87025 & 90331 \\
\hline $10 \%$ fuel (Landing) & 367 & 6.11 & 0.00 & 0.00 & 8288 & 26081 & 34368 \\
\hline 66.7\% fuel (Design Maneuver) & 4249 & 6.11 & 0.00 & 0.00 & 55251 & 173871 & 229122 \\
\hline $83.8 \%$ fuel (Design Mission) & 5341 & 6.11 & 0.00 & 0.00 & 69441 & 218524 & 287964 \\
\hline $\begin{array}{l}\text { 100\% fuel (MTOM, Ferry } \\
\text { Flight) }\end{array}$ & 6374 & 6.11 & 0.00 & 0.00 & 82877 & 260806 & 343683 \\
\hline
\end{tabular}


Table 7 MULDICON weight and balance cases, including mass moments of inertia around CG

\begin{tabular}{|c|c|c|c|c|c|c|c|c|}
\hline & \multirow[t]{2}{*}{ Weight and balance case } & \multirow[t]{2}{*}{ Mass $[\mathrm{kg}]$} & \multicolumn{3}{|c|}{ CG Coordinates } & \multicolumn{3}{|c|}{ Mass Moments of Inertia (CG) } \\
\hline & & & $X[\mathrm{~m}]$ & $Y[\mathrm{~m}]$ & $Z[\mathrm{~m}]$ & $I_{x x}\left[\mathrm{kgm}^{2}\right]$ & $I_{y y}\left[\mathrm{kgm}^{2}\right]$ & $I_{z z}\left[\mathrm{kgm}^{2}\right]$ \\
\hline \multirow[t]{6}{*}{ Gear Down } & $\mathrm{OEM}^{\mathrm{a}}$ & 6767 & 5.88 & 0.00 & 0.02 & 27438 & 20928 & 48349 \\
\hline & $\mathrm{ZFM}^{\mathrm{b}}$ & 9267 & 5.88 & 0.00 & 0.01 & 30745 & 20929 & 51656 \\
\hline & Landing $^{\mathrm{c}}$ & 7404 & 5.90 & 0.00 & 0.01 & 35726 & 23250 & 58959 \\
\hline & Design Mission $^{\mathrm{d}}$ & 14607 & 5.97 & 0.00 & 0.01 & 100186 & 40299 & 140467 \\
\hline & Ferry Flight $^{\mathrm{e}}$ & 13141 & 5.99 & 0.00 & 0.01 & 110316 & 44017 & 154314 \\
\hline & MTOM $^{\mathrm{f}}$ & 15641 & 5.98 & 0.00 & 0.01 & 113623 & 44034 & 157637 \\
\hline \multirow[t]{5}{*}{ Gear Up } & OEM & 6767 & 5.83 & 0.00 & 0.02 & 27438 & 21647 & 49068 \\
\hline & Design Maneuver ${ }^{\mathrm{g}}$ & 13516 & 5.93 & 0.00 & 0.01 & 85997 & 37133 & 123110 \\
\hline & Design Mission & 14607 & 5.94 & 0.00 & 0.01 & 100186 & 41088 & 141255 \\
\hline & Ferry Flight & 13141 & 5.96 & 0.00 & 0.01 & 110316 & 44820 & 155118 \\
\hline & MTOM & 15641 & 5.95 & 0.00 & 0.01 & 113623 & 44829 & 158432 \\
\hline
\end{tabular}

${ }^{a}$ Operational Empty Mass case: aircraft ready to fly, but without fuel or payload

bero Fuel Mass case: OEM + maximum payload

${ }^{c}$ Landing case: OEM+landing fuel

${ }^{\mathrm{d}}$ Design Mission case: OEM + mission fuel + maximum payload

${ }^{e}$ Ferry Flight case: OEM + maximum fuel

${ }^{\mathrm{f}}$ Maximum Takeoff Mass case: $\mathrm{OEM}+$ maximum fuel + maximum payload

${ }^{g}$ Design Maneuver case: $\mathrm{OEM}+$ maneuver fuel + maximum payload
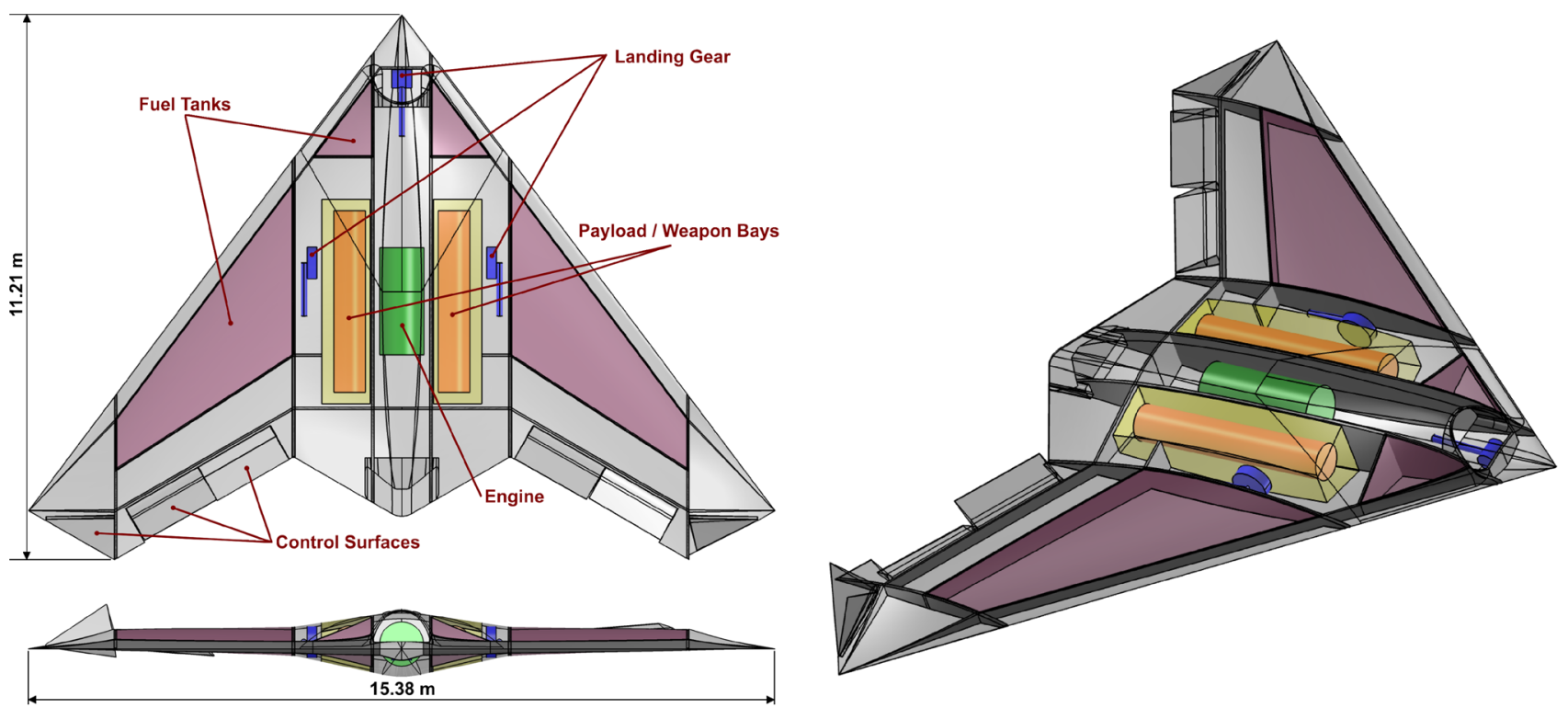

Fig. 21 3D model of MULDICON with internal arrangement 
Fig. 22 Trajectory of MULDI$\mathrm{CON}$, flying the design mission

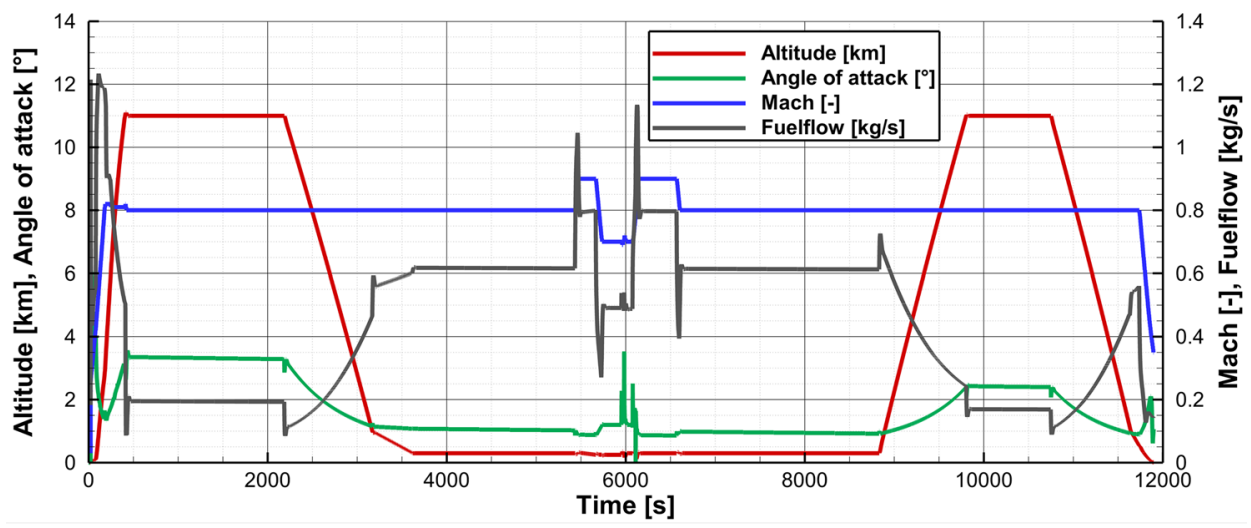

\section{References}

1. Liersch, C.M., Huber, K.C., Schütte, A., Zimper, D., Siggel, M.: Multidisciplinary design and aerodynamic assessment of an agile and highly swept aircraft configuration. CEAS Aeronaut. J. 7(4), 677-694 (2016). https://doi.org/10.1007/s13272-016-0213-4

2. Cummings, R.M., Liersch, C.M., Schütte, A.: Multi-disciplinary design and performance assessment of effective, agile NATO air vehicles. In: AIAA AVIATION Forum, American Institute of Aeronautics and Astronautics (2018). https://doi. org/10.2514/6.2018-2838

3. Cummings, R.M., Schütte, A.: An integrated computational/experimental approach to UCAV stability and control estimations: overview of NATO RTO AVT-161. In: 28th AIAA Applied Aerodynamics Conference (2010). https://doi.org/10.2514/6.2010-4392.

4. Cummings, R.M., Schütte, A.: Assessment of stability and control prediction methods for NATO air and sea vehicles. In: Final report of the AVT-161 task group RTO-TR-AVT-161 AC/323(AVT-161) TP/440. NATO RTO/AVT (2012). https://doi.org/10.14339/ RTO-TR-AVT-161

5. Cummings, R.M., Schütte, A.: Extended assessment of stability and control prediction methods for NATO air vehicles. In: Final Report of the AVT-201 Task Group STO-TR-AVT-201, NATO STO / AVT (2016). https://doi.org/10.14339/STO-TR-AVT-201

6. Liersch, C.M., Huber, K.C.: Conceptual design and aerodynamic analyses of a generic UCAV configuration. In: AIAA AVIATION Forum, American Institute of Aeronautics and Astronautics (2014). https://doi.org/10.2514/6.2014-2001

7. Cummings, R.M., Liersch, C.M., Schütte, A., Huber, K.C.: Aerodynamics and conceptual design studies on an unmanned combat aerial vehicle configuration. J. Aircr. 55(2), 454-474 (2018). https ://doi.org/10.2514/1.C033808

8. Liersch, C.M., Bishop, G.: Conceptual design of a 53deg swept flying wing UCAV configuration. In: AIAA AVIATION Forum, American Institute of Aeronautics and Astronautics (2018). https ://doi.org/10.2514/6.2018-2839

9. Department of Defense, USA: Glossary of definitions, ground rules, and mission profiles to define air vehicle performance capability. In: Military Standard. MIL-STD-3013 (2003)

10. Department of Defense, USA: Flying qualities of piloted aircraft. In: Military Standard. MIL-STD-1797A (2001)

11. Schwithal, J., Rohlf, D., Looye, G., Liersch, C.M.: An innovative route from wind tunnel experiments to flight dynamics analysis for a highly swept flying wing. CEAS Aeronaut. J. 7(4), 645-662 (2016). https://doi.org/10.1007/s13272-016-0214-3

12. Huber, K.C., Vicroy, D.D., Schütte, A., Hübner, A.R.: UCAV model design and static experimental investigations to estimate control device effectiveness and S\&C capabilities. In:
AIAA AVIATION Forum, AIAA-Paper 2014-2002, American Institute of Aeronautics and Astronautics (2014). https://doi. org/10.2514/6.2014-2002

13. Löchert, P., Huber, K.C., Liersch, C.M., Schütte, A.: Control device studies for yaw control without vertical tail plane on a $53^{\circ}$ swept flying wing configuration. In: AIAA AVIATION Forum, American Institute of Aeronautics and Astronautics (2018). https ://doi.org/10.2514/6.2018-3329

14. Schütte, A., Hummel, D., Hitzel, S.M.: Flow physics analyses of a generic unmanned combat aerial vehicle configuration. J. Aircr. 49(6), 1638-1651 (2012). https://doi.org/10.2514/1.C031386

15. Liersch, C.M., Hepperle, M.: A unified approach for multidisciplinary preliminary aircraft design. In: CEAS 2009 European Air and Space Conference (2009)

16. Liersch, C.M., Hepperle, M.: A distributed toolbox for multidisciplinary preliminary aircraft design. CEAS Aeronaut. J. 2(1-4), 57-68 (2011). https://doi.org/10.1007/s13272-011-0024-6

17. Nagel, B., Zill, T., Moerland, E., Böhnke, D.: Virtual aircraft multidisciplinary analysis and design processes-lessons learned from the collaborative design project VAMP. In: CEAS 2013 European Air and Space Conference (2013)

18. Moerland, E., Pfeiffer, T., Böhnke, D., Jepsen, J., Freund, S., Liersch, C.M., Chiozzotto, G., Klein, C., Scherer, J., Hasan, Y.J., Flink, J.: "On the design of a strut-braced wing configuration in a collaborative design environment. In: AIAA Aviation Forum 2017-17th AIAA Aviation Technology, Integration, and Operations Conference (2017). https://doi.org/10.2514/6.2017-4397

19. Boden, B., Flink, J., Mischke, R., Schaffert, K., Weinert, A., Wohlan, A., Ilic, C., Wunderlich, T., Liersch, C.M., Görtz, S., Moerland, E., Ciampa, P.D.: Distributed multidisciplinary optimization and collaborative process development using RCE. In: AIAA Aviation 2019 Forum (2019)

20. Ciampa, P.D., Nagel, B.: AGILE the next generation of collaborative MDO: achievements and open challenges. In: AIAA AVIATION 2018, Vol. paper, AIAA, Atlanta, US (2018)

21. Nagel, B., Böhnke, D., Gollnick, V., Schmollgruber, P., Rizzi, A., Rocca, G.L., Alonso, J.J.: Communication in aircraft design: can we establish a common language? In: 28th International Congress of the Aeronautical Sciences (ICAS) (2012)

22. Bachmann, A., Kunde, M., Litz, M., Schreiber, A.: A dynamic data integration approach to build scientific workflow systems. In: International workshop on workflow management (IWWM 2009), The institute of electrical and electronics engineers, Inc., pp. 27-33 (2009)

23. CPACS Homepage. URL: https://cpacs.de/. Accessed 09 Sep 2019

24. TiXI Homepage. URL: https://www.dlr.de/sc/en/desktopdefault. aspx/tabid-12766/22301_read-50944/. Accessed 09 Sep 2019 
25. TiGL Homepage. URL: https://www.dlr.de/sc/en/desktopdefault. aspx/tabid-12766/22301_read-51485/. Accessed 09 Sep 2019

26. ModelCenter Homepage. URL: https://www.phoenix-int.com/ products/. Accessed 09 Sep 2019

27. Seider, D., Fischer, P., Litz, M., Schreiber, A., Gerndt, A.: Open source software framework for applications in aeronautics and space. IEEE Aerospace Conf. (2012)

28. “RCE Homepage,” URL: https://rcenvironment.de/. Accessed 09 Sep 2019

29. Becker, R.-G., Wolters, F., Nauroz, M., Otten, T.: Development of a gas turbine performance code and its application to preliminary engine design. DLRK 2011, (2011)

30. “LIFTING_LINE Homepage," URL: https://www.dlr.de/as/en/ desktopdefault.aspx/tabid-188/379_read-625/. Accessed 09 Sep 2019

31. Horstmann, K. H.: Ein Mehrfach-Traglinienverfahren und seine Verwendung für Entwurf und Nachrechnung nichtplanarer Flügelanordnungen. Tech. rep., Deutsche Forschungs- und Versuchsanstalt für Luft- und Raumfahrt (1987)

32. Liersch, C.M., Wunderlich, T.F.: A fast aerodynamic tool for preliminary aircraft design. In: Multidisciplinary analysis and optimization conference. American Institute of Aeronautics and Astronautics (2008). https://doi.org/10.2514/6.2008-5901

33. "VSAERO User's Manual Version 7.2," Analytical Methods, Inc. (2007)

34. Voß, A.: Open and closed loop gust loads analyses for a flying wing configuration with variable longitudinal stability. Aerosp. Sci. Technol. 89, 1-10 (2019)

35. Voß, A., Klimmek, T., Bramsiepe, K.: Design, structural optimization and loads assessment for a flying wing. DLRK 2018 - Deutscher Luft- und Raumfahrtkongress (2018)

36. Bramsiepe, K., Voß, A., Klimmek, T.: Design and sizing of an aeroelastic composite model for a UCAV configuration with maneuver, gust, and landing loads. Deutscher Luft- und Raumfahrtkongress 2017 (2017)

37. Looye, G.: TECS/THCS-based generic autopilot control laws for aircraft mission simulation. In: Second CEAS specialist conference on guidance, navigation and control (2013)

38. Krüger, W., Cumnuantip, S., Liersch, C.: Multidisciplinary conceptual design of a UCAV configuration. In: RTO/AVT Panel Workshop "Virtual prototyping of affordable military vehicles using advanced MDO (2011)

39. Duus, G., Duda, H.: HAREM-Handling qualities research and evaluation using Matlab. In: IEEE International Symposium on Computer Aided Control System Design, Kohala Coast, HI (us), 22-27 August 1999, pp. 428-432 (1999)

40. Ehlers, J.: Flying qualities analysis of CPACS based aircraft models-HAREM V2.0 - In: Dlr internal report 111-2013/21, German Aerospace Center (DLR), Institute for Flight Systems (2013)

41. Löchert, P., Huber, K.C., Ghoreyshi, M., Allen, J.: Control device effectiveness studies of a $53^{\circ}$ swept flying wing configuration. Experimental, computational, and modeling considerations. Aerospace Sci. Technol. 93, 105319 (2019)

42. Löchert, P., Huber, K.C., Rütten, M.: Control concepts for an agile and highly swept flying wing configuration. Deutscher Luft- und Raumfahrtkongress 2018, DGLR (2018)

43. Schütte, A., Vormweg, J., Maye, R.G., Jeans, T.L.: Aerodynamic shaping design and vortical flow design aspects of a 53deg swept flying wing configuration. In: AIAA AVIATION Forum, American Institute of Aeronautics and Astronautics (2018). https://doi. org/10.2514/6.2018-2841

44. Kroll, N., Langer, S., Schwöppe, A.: The DLR flow solver TAUstatus and recent algorithmic developments. In: AIAA SciTech Forum, AIAA Paper 2014-080, American Institute of Aeronautics and Astronautics (2014). https://doi.org/10.2514/6.2014-0080
45. Kulfan, B.: A universal parametric geometry representation method - "CST". In: Aerospace Sciences Meetings, American Institute of Aeronautics and Astronautics (2007). https://doi. org/10.2514/6.2007-62

46. Schütte, A.: Numerical investigations of vortical flow on swept wings with round leading edges. J. Aircr. 54(2), 572-601 (2017). https://doi.org/10.2514/1.C034057

47. Zenkner, S., Becker, R.-G.: Preliminary engine design for the MULDICON configuration. In: AIAA AVIATION Forum, American Institute of Aeronautics and Astronautics (2018). https://doi. org/10.2514/6.2018-3163

48. Nangia, R.K., Coppin, J., Ghoreyshi, M.: UCAV wing design, assessment and comparisons. In: AIAA AVIATION Forum, American Institute of Aeronautics and Astronautics (2018). https ://doi.org/10.2514/6.2018-2842

49. van Rooij, M.P.C., Cummings, R.M.: Aerodynamic design of an Unmanned Combat Air Vehicle in a collaborative framework. In: AIAA AVIATION Forum, American Institute of Aeronautics and Astronautics (2018). https://doi.org/10.2514/6.2018-2840

50. Hasan, Y.J., Flink, J., Freund, S., Klimmek, T., Kuchar, R., Liersch, C.M., Looye, G., Moerland, E., Pfeiffer, T., Schrader, M., Zenkner, S.: Stability and control investigations in early stages of aircraft design. In: AIAA AVIATION Forum, American Institute of Aeronautics and Astronautics (2018). https://doi org/10.2514/6.2018-2996

51. Zenkner, S., Becker, R.-G., Trost, M., Voß, C.: Beiträge zum Triebwerksentwurf einer agilen und hoch gepfeilten Nurflügelkonfiguration. Deutscher Luft- und Raumfahrtkongress 2018, (2018)

52. Voß, C., Trost, M., Becker, R.-G.: Automated optimization of the MULDICON inlet with minimum losses and reduced sight onto the compressor front face. In: AIAA AVIATION Forum, American Institute of Aeronautics and Astronautics (2018). https://doi. org/10.2514/6.2018-3165

53. Edefur, H., Tormalm, M., Tysell, L., Quas, M.: Design and integration of a low observable intake for the MULDICON platform. In: AIAA AVIATION Forum, American Institute of Aeronautics and Astronautics (2018). https://doi.org/10.2514/6.2018-3162

54. Aref, P., Jirasek, A., Ghoreyshi, M., Cummings, R.M., Satchell, M.J.: Computational design of S-duct intakes for the NATO AVT-251 multi-disciplinary configuration. In: AIAA AVIATION Forum, American Institute of Aeronautics and Astronautics (2018). https://doi.org/10.2514/6.2018-3164

55. Schweiger, J.M., Cunningham Jr., A.M., Dalenbring, M., Voß, A., Sakarya, E.: Structural design efforts for the MULDICON configuration. In: AIAA AVIATION Forum, American Institute of Aeronautics and Astronautics (2018). https://doi. org/10.2514/6.2018-3325

56. Voß, A.: Gust loads calculation for a flying wing configuration. In: AIAA AVIATION Forum, American Institute of Aeronautics and Astronautics (2018). https://doi.org/10.2514/6.2018-3326

57. Voß, G., Schaefer, D., Vidy, C.: Investigations on flutter stability of the DLR-F19/SACCON configuration. In: AIAA AVIATION Forum, American Institute of Aeronautics and Astronautics (2018). https://doi.org/10.2514/6.2018-3327

58. Sakarya, E., Kocan, C., Okumus, B.: A study on evaluation of aeroelastic characteristics of a UCAV configuration. In: AIAA AVIATION Forum, American Institute of Aeronautics and Astronautics (2018). https://doi.org/10.2514/6.2018-3328

59. CATIA Homepage. URL: https://www.3ds.com/de/produkte-undservices/catia/. Accessed 09 Sep 2019

Publisher's Note Springer Nature remains neutral with regard to jurisdictional claims in published maps and institutional affiliations. 